\title{
Ewing sarcoma family of tumors-derived small extracellular vesicle proteomics identify potential clinical biomarkers
}

\author{
Glenson Samuel ${ }^{1,2,3, *}$, Jennifer Crow $^{4, *}$, Jon B. Klein ${ }^{5,6}$, Michael L. Merchant ${ }^{5}$ Emily \\ Nissen ${ }^{7}$, Devin C. Koestler ${ }^{3,7}$, Kris Laurence ${ }^{1}$, Xiaobo Liang ${ }^{4}$, Kathleen Neville ${ }^{8}$, \\ Vincent Staggs ${ }^{2,9}$, Atif Ahmed ${ }^{2,10}$, Safinur Atay ${ }^{4,11}$ and Andrew K. Godwin ${ }^{3,4}$ \\ ${ }^{1}$ Division of Pediatric Hematology Oncology and Bone Marrow Transplantation, Children's Mercy Hospital, Kansas City, MO, \\ USA \\ ${ }^{2}$ Department of Pediatrics, University of Missouri-Kansas City, Kansas City, MO, USA \\ ${ }^{3}$ University of Kansas Cancer Center, Kansas City, MO, USA \\ ${ }^{4}$ Department of Pathology and Laboratory Medicine, University of Kansas Medical Center, Kansas City, KS, USA \\ ${ }^{5}$ Clinical Proteomics Laboratory, Department of Medicine, University of Louisville, Louisville, KY, USA \\ ${ }^{6}$ Robley Rex VA Medical Center, Louisville, KY, USA \\ ${ }^{7}$ Department of Biostatistics \& Data Science, University of Kansas Medical Center, Kansas City, KS, USA \\ ${ }^{8}$ Department of Pediatrics, University of Arkansas for Medical Sciences, Little Rock, AR, USA \\ ${ }^{9}$ Biostatistics \& Epidemiology Core, Children's Mercy Hospital, Kansas City, MO, USA \\ ${ }^{10}$ Department of Pathology, Children's Mercy Hospital, Kansas City, MO, USA \\ ${ }^{11}$ Bristol-Myers Squibb, Cambridge, MA, USA \\ *These authors contributed equally to this work \\ Correspondence to: Andrew K. Godwin, email: agodwin@kumc.edu \\ Keywords: Ewing sarcoma; EWS-ETS; biomarkers; extracellular vesicles; exosomes \\ Received: April 18, $2020 \quad$ Accepted: June 20, $2020 \quad$ Published: August 04, 2020
}

Copyright: Samuel et al. This is an open-access article distributed under the terms of the Creative Commons Attribution License 3.0 (CC BY 3.0), which permits unrestricted use, distribution, and reproduction in any medium, provided the original author and source are credited.

\section{ABSTRACT}

Purpose: Ewing Sarcoma Family of Tumors (ESFT), the second most common pediatric osseous malignancy, are characterized by the pathognomonic chromosomal EWS-ETS translocation. Outside of tumor biopsy, no clinically relevant ESFT biomarkers exist. Additionally, tumor burden assessment at diagnosis, monitoring of disease responsiveness to therapy, and detection of disease recurrence are limited to radiographic imaging. To identify new, clinically relevant biomarkers we evaluated the proteome of a subset of ESFT-derived small extracellular vesicles (sEVs).

Materials and Methods: We performed the first high quality proteomic study of ESFT-derived $s E V s$ from 5 ESFT cell lines representing the most common EWS-ETS fusion types and identified 619 proteins composing the core ESFT sEV proteome. We compared these core proteins to databases of common plasma-based proteins and sEV-associated proteins found within healthy plasma to identify proteins unique or enriched within ESFT.

Results: From these analyses, two membrane bound proteins with biomarker potential were selected, CD99/MIC2 and NGFR, to develop a liquid-based assay enriching of ESFT-associated SEVs and detection of SEV mRNA cargo (i.e., EWS-ETS transcripts). We employed this immuno-enrichment approach to diagnosis of ESFT utilizing plasma $(250 \mu \mathrm{l})$ from both localized and metastatic ESFT pediatric patients and cancer-free controls, and showed significant diagnostic power [AUC $=0.92$, $p=0.001$ for $s E V$ numeration, with a PPV $=1.00,95 \% \mathrm{CI}=(0.63,1.00)$ and a NPV $=0.67,95 \%$ CI $=(0.30,0.93)]$.

Conclusions: In this study, we demonstrate utilization of circulating ESFTassociated SEVs in pediatric patients as a source of minimally invasive diagnostic and potentially prognostic biomarkers. 


\section{INTRODUCTION}

Ewing Sarcoma Family of Tumors (ESFT) encompass a group of highly aggressive pediatric osseous and soft tissue malignancies thought to originate from primordial bone marrow-derived mesenchymal stem cells and consists of small round blue cells with minimal stroma and differentiation [1]. Ewing sarcoma of the bone, extraosseous Ewing sarcoma, and peripheral primitive neuroectodermal tumors (pPNET) are all considered manifestations of a single neoplastic entity. With a peak incidence within the second decade of life, slight male preponderance, high incidence in those of European ancestry and approximately 3 cases/million/year [2], this malignancy continues to remain the second most prevalent pediatric bone tumor after osteosarcoma. ESFT can occur in any bone; however, most typical sites of involvement are the pelvis (25\%), femur (16\%), ribs (13\%), spine (8\%), and scapula (5\%) [3]. Overt metastatic disease is prognostic, with approximately $25 \%$ of newly diagnosed ESFT being affected [4]. Of the ESFT metastatic patients, $37 \%$ (or $9 \%$ of all ESFT patients) have metastases confined to the lung or pleura [5].

Overt metastatic disease is prognostic; however, it is evident that the preponderance of ESFT patients (even with localized disease) harbor micrometastatic disease. Hence, the necessity for a multidisciplinary approach in the management of these patients, involving interval compression chemotherapy with that of local control (surgical and/or radiation) as well. Localized ESFT patient outcomes based on the most recent Children's Oncology Group completed trial (AEWS0031) is 73\% Event Free Survival (EFS) at 5 years [6]. Outcomes continue to remain dismal for pediatric metastatic ESFT, with a $20-30 \%$ 2- to 3-year EFS. Despite the intensification of therapies (interval-compressed VDC/IE) and improved local control in pediatric ESFT, $30-40 \%$ of patients experience recurrence [7]. Most recurrences occur within 2 years from time of diagnosis, with an EFS of less than $10 \%$ at 3 years for early recurrence ( $<2$ years from diagnosis) [8] and for late recurrences ( $>2$ years from diagnosis) greater than $25 \%$ OS (overall survival). The median relapsefree interval (time of diagnosis to first recurrence) is 17 months (range 5-90 months) [5]. Outside of the presence of overt metastatic disease, no clinically relevant predictive biomarkers exist which are indicative of the increased risk of recurrence in localized ESFT patients. Various other clinical prognostic factors such as age ( $>15$ years of age with worse outcomes), tumor location (pelvic tumors with worse outcomes) [6], tumor size (larger tumors $>100$ $\mathrm{mL}$ with worse outcomes) [9] and histologic response to induction therapy (poor response with worse outcomes) [10]; however, have not been incorporated within pediatric ESFT treatment risk stratification [11]. Standard circulating tumor markers are not applicable to ESFT. Serum lactate dehydrogenase (LDH) has been most consistently associated with aggressive disease, but lacks ESFT specificity [12].
The clinical presentation of ESFT are often nonspecific in nature, with pain, swelling and discomfort consisting of the most typical complaints and are all related to growth of these tumors. According to the literature, there typically is a lapse of three to nine month range from onset of symptoms prior to time of initial diagnosis, thus delaying initiation of oncological management [5]. Currently, diagnostic and monitoring modalities for children and young adults with ESFT require utilization of radiographic imaging and the important diagnostic testing via biopsies of suspicious lesions for establishment of diagnosis and prognosis in these patients. Although the standard of care, these tests are expensive, invasive and associated with potential long-term risks. Traditionally, ESFT was considered a diagnosis of exclusion, but over the past few decades, with the introduction of immunohistochemical markers, e.g., CD99/MIC2 and detection of the oncogenic chimeric fusion involving the Ewing sarcoma RNA (ribonucleic acid) binding protein 1 gene (EWSR1 gene; Ewing sarcoma breakpoint region 1) [13], which is a hallmark of ESFT, the accuracy of diagnosis has considerably improved. However, these approaches require invasive open or core biopsy sampling of active tumor tissue [14]. The most utilized immunohistologic stain in ESFT diagnosis is the monoclonal antibody CD99 (MIC-2), which recognizes the cell surface protein. ESFT specimens demonstrate a crisp and strong membranous positivity with CD99 antibody in more than $90 \%$ to $95 \%$ of cases reported. Therapeutic response assessment is based upon tumor size changes as determined with anatomic imaging tests. Utilization of FDG PET-CT in staging, restaging and assessment of response to ESFT therapy is increasing worldwide although not considered a standard in the diagnostic workup [15]. Even children and young adults successfully treated for their localized ESFT, are at high risk of relapse, and must be monitored for years by periodical medical imaging examinations, often resulting in additional X-ray exposure. Absence of asymptomatic ESFT diagnostic biomarkers has lent to the reliance on clinical symptomatology and/or findings with complementary conventional imaging modalities including FDG PET-CT, to detect and monitor these patients. However, imaging in and of itself is a poor means for early cancer detection and monitoring of recurrence. Therefore, the discovery of new ESFT biomarkers and development of clinically useful tests for early detection and monitoring disease progression are considerably in need.

There has been a momentum towards the direction of personalized medicine, especially in solid pediatric tumors such as ESFT and other pediatric sarcomas [16]. It naturally follows that the identification of novel and robust biomarkers as well as the tools to effectively measure them are in dire need. Many of the biomarkers studied regarding ESFT have been prognostic in nature and rely upon biopsy/resection of tumor tissue [17, 18]. Currently, there are no readily available clinical liquid-based assays 
utilizing biological fluids such as blood, serum, or urine specifically for diagnosing ESFT, evaluating minimal residual disease, or monitoring of disease progression [4].

To address some of these diagnostic hurdles, we turned our attention to a class of circulating extracellular vesicles (EVs), of which small EVs (sEVs) or exosomes have gained considerable traction in the field of liquid-based biomarkers. sEVs/exosomes are proving to be an abundant source of protein- and nucleic acid-related biomarkers [19-22]. Exosomes originate through the formation of multivesicular bodies (MVB) within the endosomal compartment of cells [23] and are secreted into the extracellular space as a result of fusion with the cellular plasma membrane. sEVs contain a varying assortment of proteins, lipids, and nucleic acids reflective of their cell of origin. The population of sEVs within the blood is heterogenous because circulating extracellular vesicles are released by most if not all types of cells in the body. It is estimated that exosomes released by platelets, lymphocytes, dendritic cells, and other immune cells comprise $80-90 \%$ of serum/plasma exosomes [24]. In contrast to other classes of extracellular vesicles, tumor derived $\mathrm{sEV}$ s/exosomes, have been found to be elevated within the circulation of cancer patients and reflective of their tumor burden $[25,26]$. The cell specific cargo of sEVs, including a wide array of proteins and RNAs (e.g., mRNA, miRNA, and LncRNA), has been shown to have biomarker potential in several malignancies including; gastrointestinal stromal tumor [25], pancreatic cancer [27], acute myeloid leukemia [28] and glioblastoma multiforme [29]. In addition, our group and others, have shown that tumor derived sEVs play pivotal roles in intercellular communication, tumor development, angiogenesis [30], preparation of pre-metastatic niches [31], modulating antitumor immune responses [32] and drug resistance [33]. Tumor derived sEVs are found abundantly circulating in blood plasma and within malignant effusions derived from cancer patients $[34,35]$. Furthermore, previous work has suggested that tumor derived SEVs may provide a biomarker source for ESFT [36]. The essential role of EWS-ETS fusion transcripts makes it less likely to be down-regulated or nonexistent during tumor progression, convincingly supporting their routine utility for diagnostic assessment in ESFT tumor biopsied tissues but also in circulating tumor derived sEVs. In this study we report for the first time the proteome of ESFT-derived sEVs/exosomes and develop a clinically useful test based on immuno-enrichment of ESFT-sEVs and detection of the EWS-ETS fusion transcripts.

\section{RESULTS}

\section{Ewing sarcoma family of tumor cell lines constitutively release EWS-ETS transcript and oncoprotein in association with SEVs}

For this study, ESFT cell lines representative of the most common EWS-ETS fusion types; namely EWS-
FLI1 type I (TC-71), EWS-FLI1 type II (RD-ES and SKES-1), EWS-FLI1 type III (CHLA-258) fusions, as well as COG-E-352 which carries the EWS-ERG fusion were used as sources for sEVs. In addition to these ESFT cell lines, Hs919. T, a benign osteoid osteoma cell line known to lack EWS-ETS fusions was included as a negative control (Supplementary Table 1) [37]. sEVs from each of the ESFT and control cell lines were isolated using ultracentrifugation [38] and the contents of the resulting $120,000 \times$ g pellet were then characterized for markers and size distribution (Figure 1 and Supplementary Figure 1). Nanoparticle Tracking Analysis (NTA) indicated that all five ESFT cell lines released a homogenous mixture of nano-sized vesicles with varying diameters between 150-239 nm (Supplementary Figure 1), consistent with previous reports for sEVs [39]. In addition, western blot analysis confirmed an enrichment of exosomal markers, Alix and CD81, in sEVs as compared to the parental cellsderived lysates (Figure 1A), further supporting the purity of the isolated sEVs. Using a monoclonal antibody raised against the C-terminal domain of FLI1, the expression of the EWS-FLI1 fusion protein was also only detectable in sEVs derived from cell lines carrying the EWS-FLI1 fusion transcripts (Figure 1A), while COG-E-352 (EWS$E R G)$ and Hs919. T cell line derived sEVs were negative. Of note, size differences in EWS-ETS oncoproteins were noticeable between cellular extracts and their corresponding sEV lysates, suggesting these fusion proteins and other $\mathrm{sEV}$ proteins may be post-translationally modified prior to sorting into sEVs as previously been described [40]. Next RT-PCR analysis demonstrated and validated that ESFT cell lines known to have an EWS-ETS fusion were positive for either the EWS-FLII or EWS-ERG transcripts (Figure 1B). Most importantly, enrichment of the EWS-ETS transcripts were detectable within each of the ESFT cell line derived sEVs while absent within Hs919. T cells-derived sEVs (Figure 1C). In addition to PCR we evaluated the mRNA content of both cell lysates and their corresponding sEVs using a portion of the Nanostring Elements sarcoma panel as previously published [41]. While we were able to detect and differentiate EWS-ETS fusion transcripts in cellular mRNA, we were unable to detect the presence of the fusion transcript within sEV samples using the nCounter platform (Nanostring) and a custom designed EWS-ETS fusion transcript panel (data not shown), which led us to choose qPCR as our primary assay for transcript detection. Taken together these data demonstrate that sEVs released from ESFT cell lines harbor the pathognomonic EWS-ETS fusion protein and transcript characteristic of ESFT.

\section{Proteomic analysis of ESFT cell lined derived sEVs identify a core set of ESFT-associated exo- proteins}

Two biological replicates of ESFT sEV preparations were isolated from TC-71 and CHLA-258, while one was 
isolated from RD-ES, SK-ES-1, and COG-E-352 cell lines derived conditioned media. Each biological replicate was analyzed as technical replicates to further establish a reliable sEV proteome (i.e., exo-proteome) profile.

Our initial analysis of ESFT cell line derived $\mathrm{sEV}$ proteomic data utilizing the Proteome Discoverer v1.3.0.330 (Supplementary Table 2) we determined the qualitative presence and distribution of identified proteins (total 1,082 proteins) between the five pediatric ESFT cell line derived sEVs, revealing a common subset of 619 sEV proteins out of 822 (TC-71), 870 (SK-ES-1), 876 (COG-E-352), 914 (CHLA-258), and 1,009 (RD-ES) unique peptides, representing an $75 \%, 71 \%, 71 \%, 68 \%$, and $61 \%$ overlap, respectively (Figure 2 ). This observation was further strengthened, i.e., of the total proteins $(1,082)$ identified between the five cell lines, only 5 proteins $(0.5 \%)$ were enriched solely in TC-71, $7(0.6 \%)$ in SKES-1, $9(0.8 \%)$ in COG-E-352, 28 (2.6\%) in CHLA-258, and $54(5 \%)$ in RD-ES sEVs (Figure 2).

Analysis of the mass spectrometry proteomic data from sEVs derived from ESFT cell lines, EWS-FLI1 Type I (TC-71, $n=2$ ), EWS-FLI1 Type II (SK-ES-1, $n=1$ and RD-ES, $n=1$ ), EWS-FLI1 Type III (CHLA258, $n=2)$ and EWS-ERG (COG-E-352, $n=1)$ were analyzed utilizing the total averaged spectrum counts (Supplementary Table 3) for differential protein expression comparison analysis. For EWS-FLI1 Type I versus Type II a differential expression in 437 proteins was noted, while 322 proteins in EWS-FLI1 Type I vs Type III, 241 proteins in EWS-FLI1 Type II vs Type III, and 572 for EWS-FLI1 vs EWS-ERG. (Supplementary Table 4 and Figure 3).

\section{Pathway analysis of proteomic data}

Mass spectrometry proteomic data via the Proteome Discoverer v1.3.0.330 was utilized to gain an understanding of the origin of the core ESFT sEV proteome using the Functional Enrichment analysis tool (FunRich). Unsurprisingly, the largest cellular component of origin was listed as 'extracellular exosomal' ( $\sim 80 \%)$ (Supplementary Figure 2A). Many of the shared ESFT $\mathrm{sEV}$ proteins were found to be ubiquitously exosomal proteins, such as membrane transport and fusion proteins (Annexins A1, A11, A2, A5, A6, A7), tetraspanin protein CD81, chaperone proteins (heat shock family protein 70, HSP70 and heat shock protein family 90, HSP90), metabolic enzymes (pyruvate kinase, ATPase, G6Pisomerase, glyceraldehyde 3-phosphate dehydrogenase,

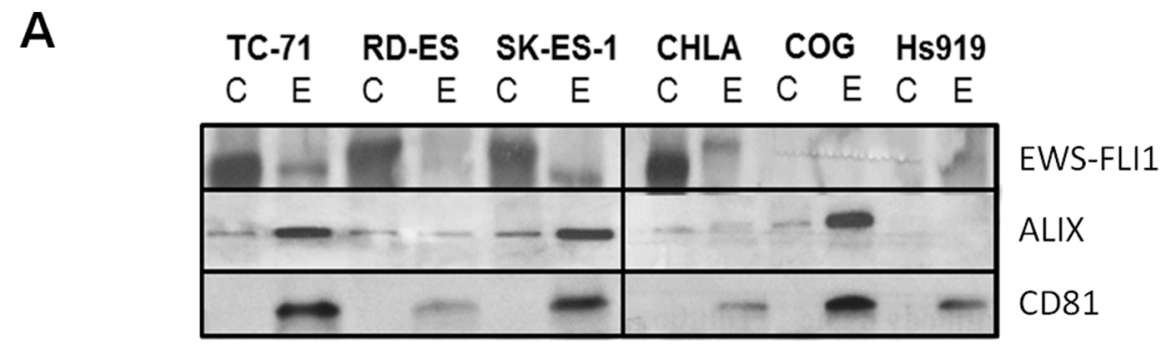

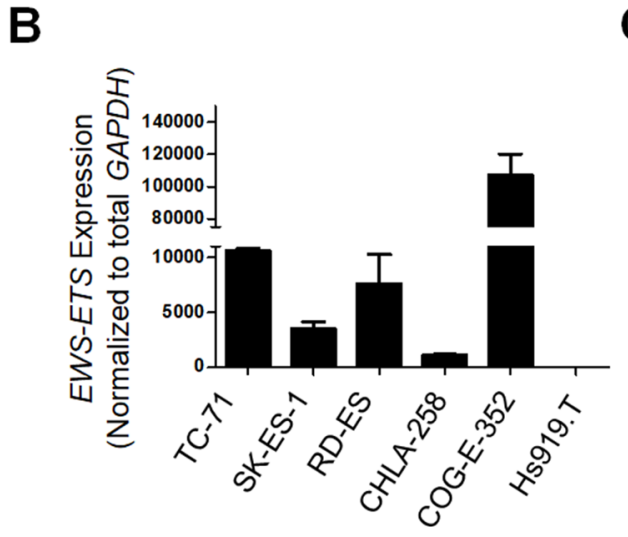

Cell Lines
C

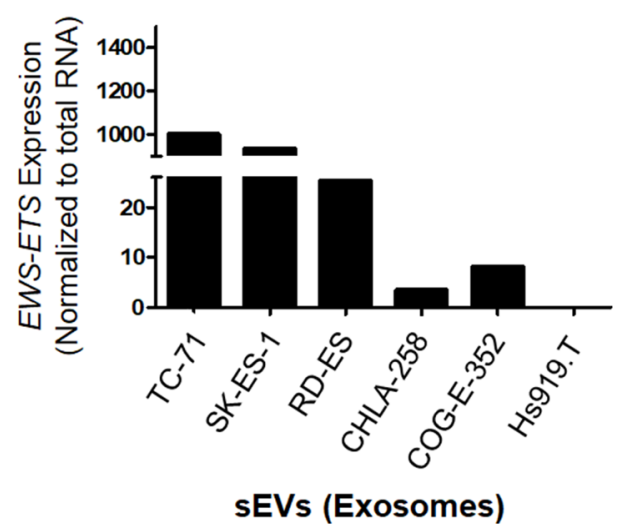

Figure 1: EWS-ETS transcript and protein are detected in ESFT cell lines and their corresponding $\mathbf{s E V s}$. (A) Western blot analysis of EWS-FLI1 protein and common exosomal markers (ALIX, CD81) in ESFT cell lines (C) and sEVs (E) from TC-71, RD-ES, SK-ES-1, CHLA-258, and COG-E-352 and Hs919.T, a benign osteoid osteoma cell line, was used as a negative control. EWSFLI1 is detected using an antibody directed at the C-terminal region of FLI1. ALIX and CD81 common markers of exosomal and small EV populations. (B) Expression of the EWS-FLII or EWS-ERG fusion transcripts in ESFT and Hs919.T (negative control) cell lines. Expression values were normalized to the housekeeping gene GAPDH. (C) Expression levels of EWS-FLI1 or EWS-ERG fusion transcripts in ESFT cell line derived sEVs normalized to total RNA input. 
enolase, aldolase, phosphoglycerate kinase 1), antigen presenting proteins (MHC-1, H2A and complement), and cytoskeletal structural proteins (ARP2, ARP3, cofilin 1 , moesin, actin gamma 1, syndecan binding protein) (Supplementary Figure 2).

To gain a further understanding of the functional classification of ESFT sEV proteins, we used Protein ANalysis THrough Evolutionary Relationships [42] 10.0 Gene ontology Molecular Function software. This software allowed the identification of top molecular functions, biological processes, and protein classes within our ESFT sEV proteome. We identified 288 different molecular functions with the uppermost consisting of protein binding, catalytic activity, and structural molecule activity (Supplementary Figure 2B). Out of 550 identified cellular processes, proteins involved within the metabolome, cellular component organization and biogenesis, biological regulation, localization processes, and response to stimulus were most enriched in ESFT derived sEVs (Supplementary Figure 2C). The bulk of protein class consisted of nucleic acid binding, hydrolase, enzyme modulator, cytoskeletal protein, and chaperone proteins (Supplementary Figure 2D). This analysis demonstrates that ESFT derived sEVs carry a wide variety of proteomic content composed of both common sEVs elements and others which may be more specifically characteristic of ESFT.
To identify disrupted biological pathways between EWS-FLI1 versus EWS-ERG cell line derived sEVs, a gene set enrichment analysis (GSEA) was implemented. This analysis demonstrated two statistically significant pathways enriched between the comparison groups, the ECM-receptor interaction ( $p$-value: 0.012) and the focal adhesion pathway ( $p$-value: 0.026) (Supplementary Table 5).

\section{Identification of candidate ESFT sEVs proteins as potential biomarkers}

Subsequent to this proteomic analysis, we then asked whether any of these ESFT sEV-proteins could serve as potential ESFT biomarkers and be exploited to specifically enrich for circulating tumor-derived sEVs. The human plasma constitutes of approximately $7 \%$ proteins and considered the most complex humanderived proteome containing other tissue proteomes as subsets [43]. To reach the full potential of ESFT derived sEVs as a source of biomarkers and distinguish their proteome from contaminating plasma proteins, it is essential to differentiate specific ESFT derived sEV proteins applying a large robust normal/healthy proteome dataset. The Plasma Proteome Project (http://www. plasmaproteomedatabase.org/) is the characterization of

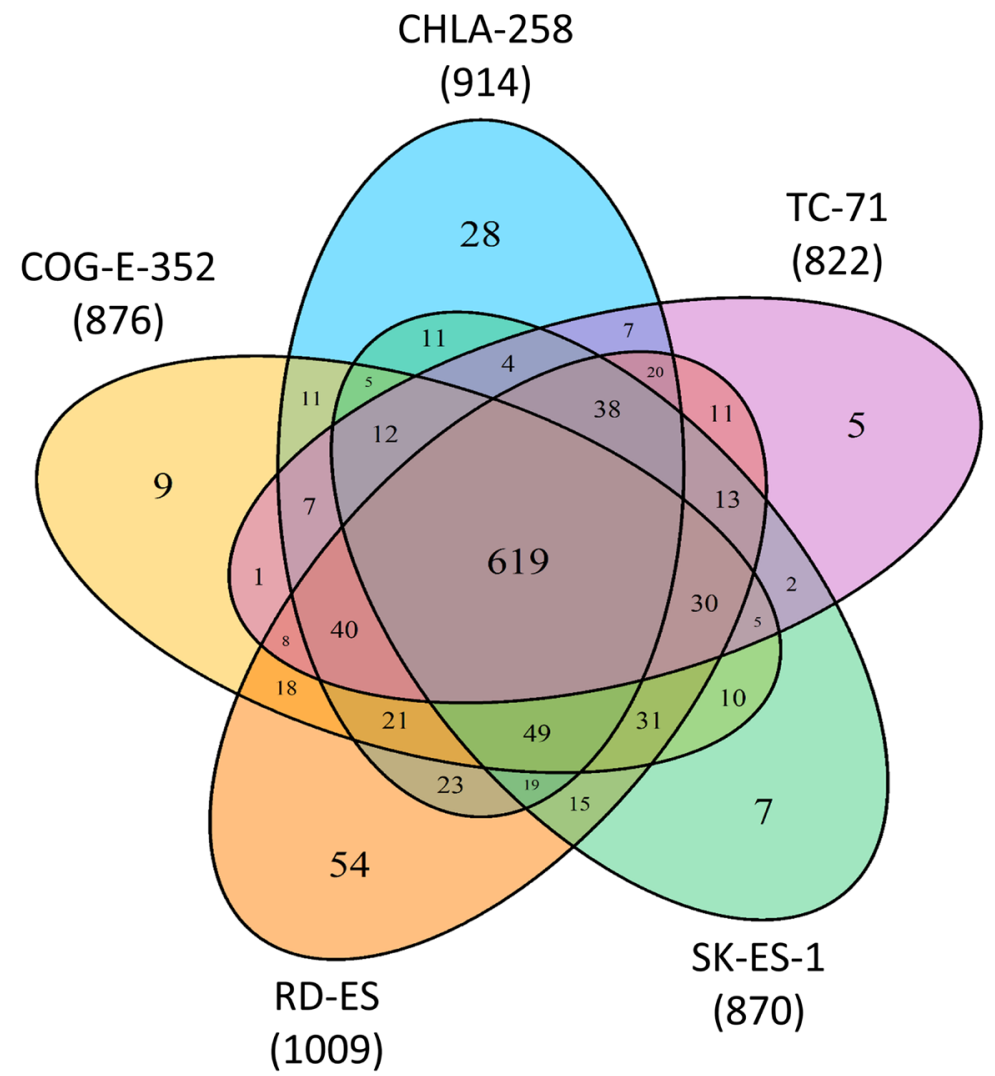

Figure 2: Common and unique proteins to ESFT cell line derived sEVs. Venn diagram depicting quantitative measurements from spectrometry analysis of 5 Ewing Sarcoma Family of Tumors (ESFT) cell line (EWS-FLI1 fusion Type I, II, III, and EWS-ERG fusion) derived sEVs. 
the human plasma proteome by an international consortium and is one of the largest resources of proteomics reported. All proteins previously reported to be found within the plasma and serum of healthy individuals were eliminated by manually comparing our initial proteomic ESFT cell line derived sEV dataset (Proteome Discoverer v1.3.0.330 based data set) to that of the proteins in the Plasma Proteome Database. This approach identified a total of 60 potential ESFT biomarker candidates. Even though present in ESFT-derived sEVs, CD99/MIC2 and HINT1 were overlooked based on this type of analysis. However, given their documented importance related to ESFT (discussed below) we reincorporated them onto the candidate list resulting in a total of 62 protein biomarkers (Supplementary Table 6).

We then utilized the Ingenuity Pathway Analysis (IPA) software platform for gene ontology and pathway analysis to elucidate biomarkers previously published within sarcomas (QIAGEN Inc., https://www. qiagenbioinformatics.com/products/ingenuitypathwayanalysis). IPA is a curated database that utilizes current knowledge available on genes, proteins, normal cellular and pathological processes, signaling and metabolic pathways, required for pathway construction. We utilized the Ingenuity Biomarker Analyzer tool, to identify cellular biological function and canonical pathways in known proteins previously identified as biomarkers. Present within this data set were immuno-histochemical markers commonly expressed in ESFT, e.g., CD99/MIC2 and CAV1 and are used clinically to differentiate ESFTs from other small round cell tumors. Another marker identified was Histidine triad nucleotide binding protein 1 (HINT1). HINT1 has been shown to repress $\beta$-catenin-mediated transcription of Wnt target genes and had been noted to be differentially expressed between localized and metastatic ESFT [44]. All together a total of 10 proteins previously identified to be associated with sarcomas were further investigated (Supplementary Table 7 and Figure 4A).

Our next step was to then confirm the presence and enrichment of these proteins within our cell lines and cell line-derived sEVs by western blot analysis (Figure 4B and 4C). This analysis corroborated that CD99 and NGFR were expressed within ESFT cell lines and enriched within ESFT-derived sEV. The protein levels were significantly lower or absent in the control Hs919. T cells and its associated sEVs (Figure 4B). Likewise, HINT1, EZR, and ENO were enriched in a majority of ESFT sEV samples and minimally enriched or absent in control (Figure 4B and 4C). ITGA5 (Integrin Subunit Alpha 5), JAK1 (Janus Kinase 1), NPM1 (Nucleophosmin), and CTNNB1 (Catenin Beta 1) were either not detected in ESFT or not substantially enriched in ESFT samples as compared to the control (Figure 4C). Taken together, this analysis lead towards the identification of 5 proteins, $(2$
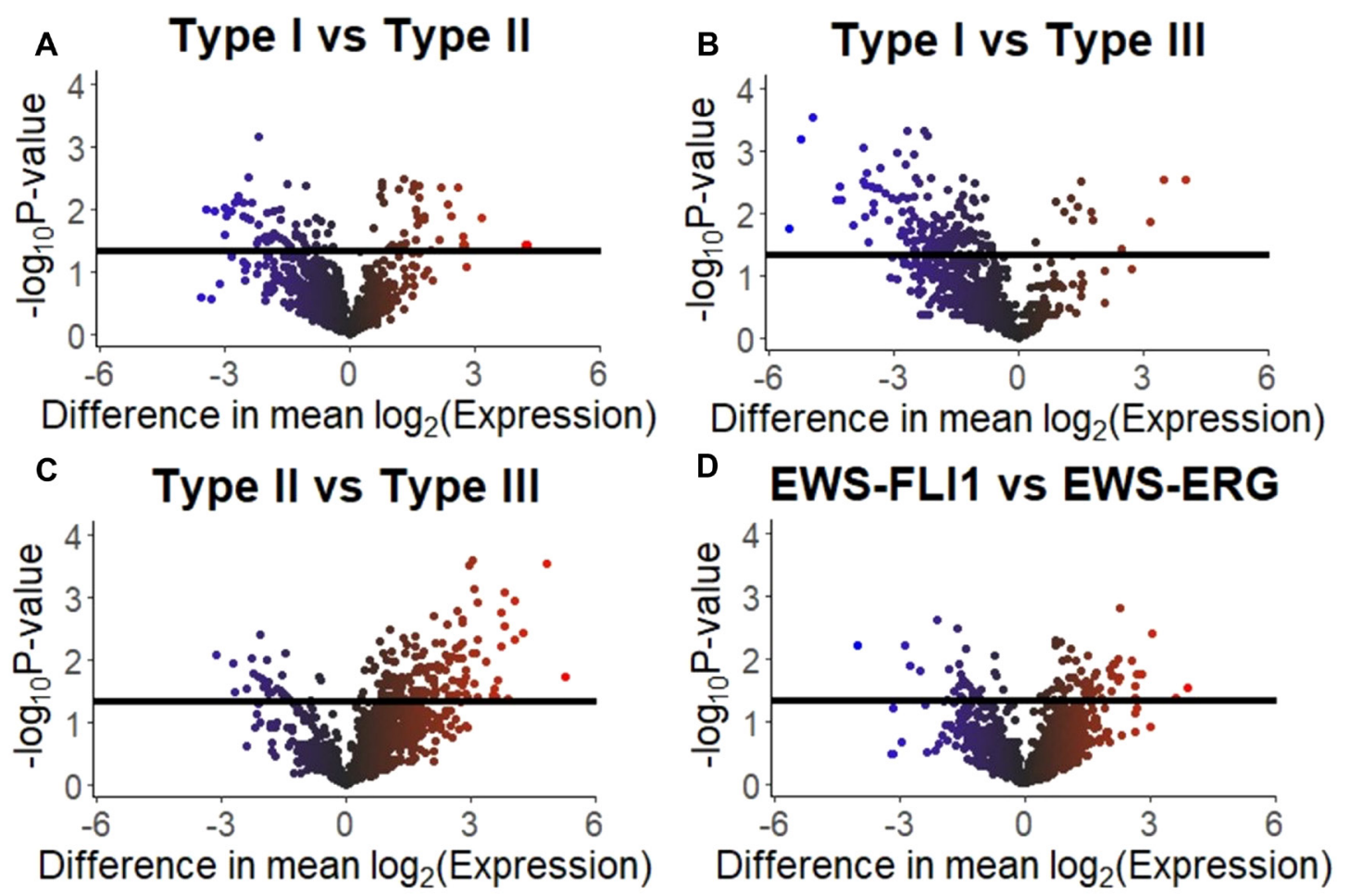

Figure 3: Differentially expressed proteins in ESFT cell line derived sEVs. Volcano plots visualizing the results of the protein expression analysis based on the four comparisons of interest: (A) Type I versus Type II fusions, (B) Type I versus Type III fusions, (C) Type II versus Type III fusions, and (D) EWS-FLI1 versus EWS-ERG. Horizonal black bars represent a $p$-value of 0.05 [e.g., -log10(0.05)]. The color of the plotted protein represents the difference in mean expression. The scale is -6 to 0 to 6 with the color scale as blue to black to red, respectively. 
membrane bound and 3 cytosolic) with the potential as ESFT sEV biomarker capability. We next evaluated the two most promising membrane-associated biomarkers (NGFR and CD99/MIC2) by IHC on primary tumor biopsies. Over $90 \%$ of tumor biopsies expressed high levels for membranous CD99. For NGFR, over 50\% of tumors stained between medium and high levels and less than $18 \%$ were negative for the marker (Supplementary Figure 3).

\section{Immunoprecipitation with NGFR and CD99 enriches for tumor derived sEVs}

To begin to develop a clinical assay based on circulating tumor derived sEVs, we opted to exploit the membranous location of CD99 and NGFR to develop a tool capable of enriching ESFT-associated sEVs from plasma in pediatric patients. Our group as well as others have attempted to detect exosomal EWS-ETS transcripts directly from plasma, but have been relatively unsuccessful. To begin, we determined the minimal plasma volume required for $\mathrm{sEV}$ detection in order to best minimize the risks of hemodynamic instability or iatrogenic anemia within our pediatric patient population. We isolated sEVs from 250 $\mu \mathrm{L}$ and $500 \mu \mathrm{L}$ of plasma from 3 healthy individuals using ultracentrifugation $(120,000 \times \mathrm{g}$ pellet $)$ and then isolated total RNA from the resulting pellet. The amount of total RNA was compared to RNA isolated from a control volume $(5 \mu \mathrm{g})$ of ESFT cell-derived sEVs (Figure 5A). From this we determined that $250 \mu \mathrm{l}$ would be a sufficient volume for sEV RNA isolation and subsequent evaluation of EWSETS fusion transcripts. Next, we utilized healthy control plasma samples spiked with ESFT cell line derived sEVs (Figure 5B). $1 \mathrm{~mL}$ of plasma from healthy control samples was spiked with an excess of TC-71 derived sEVs $(1 \times$ $10^{10}$ ). From this we immunocaptured (IP) EVs from $250 \mu \mathrm{L}$ aliquots using CD99 or NGFR antibodies alone or in 50/50 combination (combo) and with $\mathrm{IgG}$ as a negative control (NC) (Figure 5B). We observed little to no difference in quantification of EVs enriched from plasma with either the single antibodies or combination (combo) (Figure 5C). However, under these conditions we observed substantial non-specific binding of sEVs to the beads with IgG alone despite multiple attempts at different blocking techniques. Even given this hurdle, we obtained 1-3 $\mu \mathrm{g}$ of total RNA from sEVs isolated using either individual antibody or combo (Figure 5D). Real-time qPCR revealed that IPs with CD99, NGFR, and combo captured EVs harboring the EWSETS fusion transcript (Figure 5E). Given these data, and to ensure that we were optimizing our ESFT sEV enrichment, we opted to utilize the combo IP technique for subsequent studies. To note: EVs will be used in reference towards all particles isolated via IP method due to the IP technique itself is incapable of enriching only sEV populations.

\section{Identification of EWS-ETS transcripts from clinical samples}

To transition our assay into a pre-clinical application, we isolated EVs using immuno-enrichment

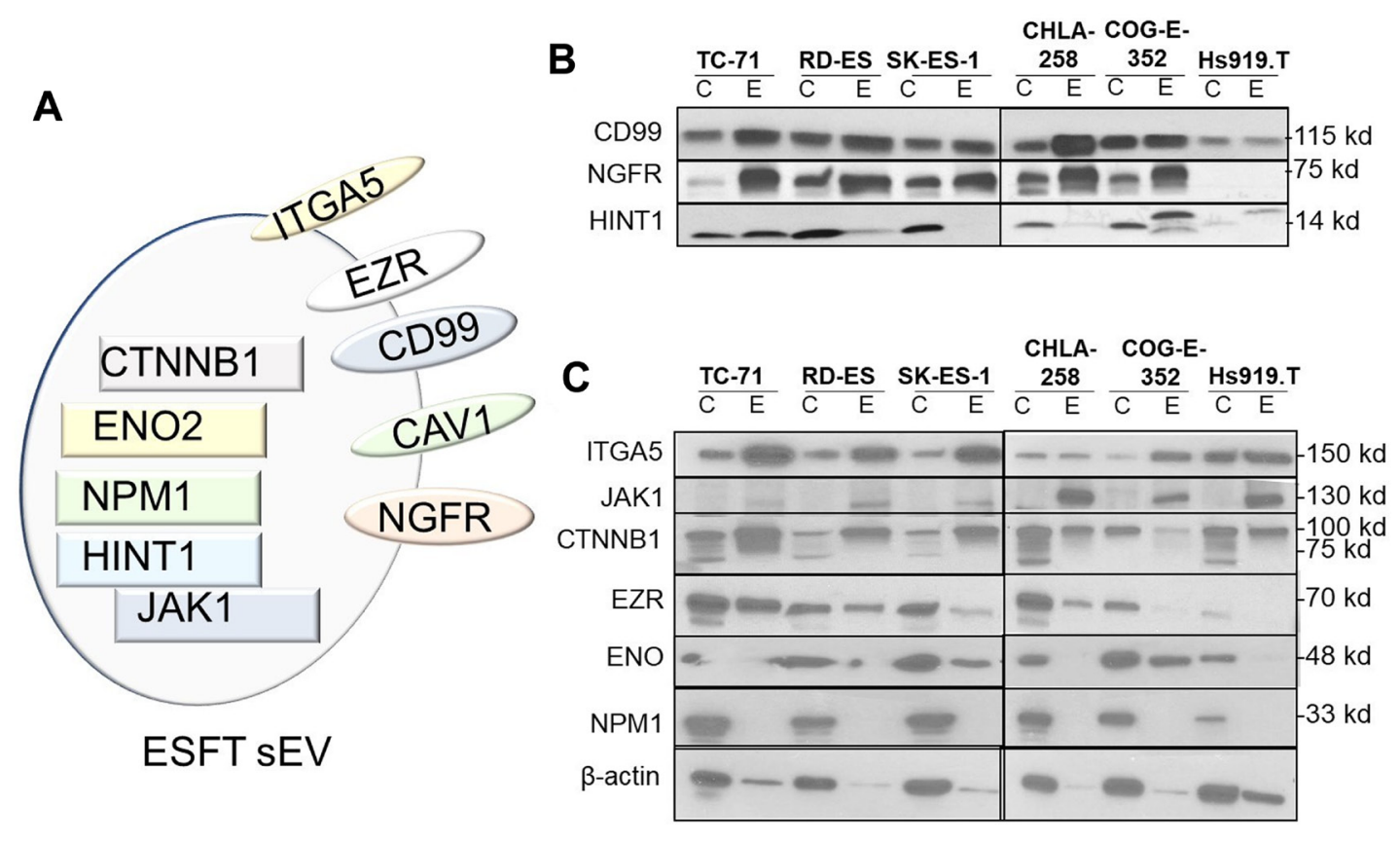

Figure 4: Identification of potential sEV-associated ESFT biomarkers. (A) Cellular localization of potential ESFT sEV biomarkers (Intraluminal, rectangles; plasma membrane, ovals). (B-C) Validation of ITGA5 (Integrin Subunit Alpha 5), JAK1 (Janus Kinase 1), CTNNB1 (Catenin Beta 1), EZR (Ezrin), ENO (Enolase), and NPMN1 (Nucleophosmin 1) as potential sEV-protein biomarkers for ESFT by western blot analysis. 
from 10 clinical pediatric ESFT plasma samples (ages 1 to 17 years of age) and 6 plasma samples from healthy control individuals ( $<20$ years of age) (Table 1$)$. From $250 \mu \mathrm{L}$ of healthy patient plasma we immunoprecipitated an average of $2 \times 10^{8} \mathrm{EVs}$. In comparison, we isolated $\sim 16$-fold more EVs (average $32 \times 10^{8} \mathrm{EVs}, P=0.01$ ) utilizing the equivalent volume of ESFT pediatric patient plasma (Figure 6A). This was true for both subsets of ESFT patients, localized $(P=0.01)$ and metastatic $(P=0.04)$ disease. However, there was no significant variance between the number of EVs in ESFT patients with localized vs. metastatic disease. ROC/AUC analysis of these data resulted in a $95 \%$ confidence interval with an AUC of 0.9242 (Figure 6B). Using qRT-PCR analysis of the nucleic acid content of the isolated EVs by IP, we identified the EWS-ETS fusion transcript in 70\% (7 of $10)$ of pediatric clinical samples $(60 \%$ ESFT metastatic and $83 \%$ ESFT localized identified) (Figure 6C), with no false positives. cDNA from EVs derived from TC71 and Hs919. T cell lines were used for positive and negative controls, respectively. These results equated to a positive predictive value (PPV) of $1.00(0.63,1.00)$ and negative predictive value (NPV) of $0.67(0.30,0.93)$ for detecting the EWS-ETS fusion transcript. To evaluate if this methodology is more efficacious than evaluating EVs as a whole, we prepared matched samples from 4 ESFT pediatric patients using immuno-isolation with combo (CD99+NGFR) or a member of the tetraspanin superfamily CD9 (a common sEV marker) in $250 \mu \mathrm{L}$ of plasma. While no significant differences in the number of
EVs were isolated (Supplementary Figure 4A), we were able to identify the EWS-ETS transcript in all of the ESFT patients using the COMBO immuno-isolation approach, but only $50 \%$ of the patients using CD9 antibody alone (Supplementary Figure 4B). These pre-clinical data are strongly supportive of the approach to use CD99 and NGFR for the enrichment for ESFT specific EVs from clinical patient plasma and that the proposed liquidbase biopsy can serve as a clinical tool for the diagnosis, monitoring of disease, and early detection of relapse (Figure 6D).

\section{DISCUSSION}

As with the majority of pediatric malignancies, translational research in ESFT lags behind work being done in adult oncology. In this study, we have sought to advance the scientific literature of ESFT by defining for the first time the proteome of ESFT-associated sEVs. The idea of a "liquid biopsy" has prompted a plethora of studies on sEV biomarkers in cancer [45]. However, isolation of tumor-associated sEVs directly from patient blood samples is particularly challenging, in part, due to lack of specific markers capable of distinguishing cancer from non-cancerous derived sEVs. We hypothesized that the we could utilize protein biomarkers towards the enrichment of ESFT-associated $\mathrm{sEVs}$, given the EWSETS fusion transcript is not readily detected in plasma. Additionally, we speculated that, in doing this, we could significantly increase the sensitivity in detection
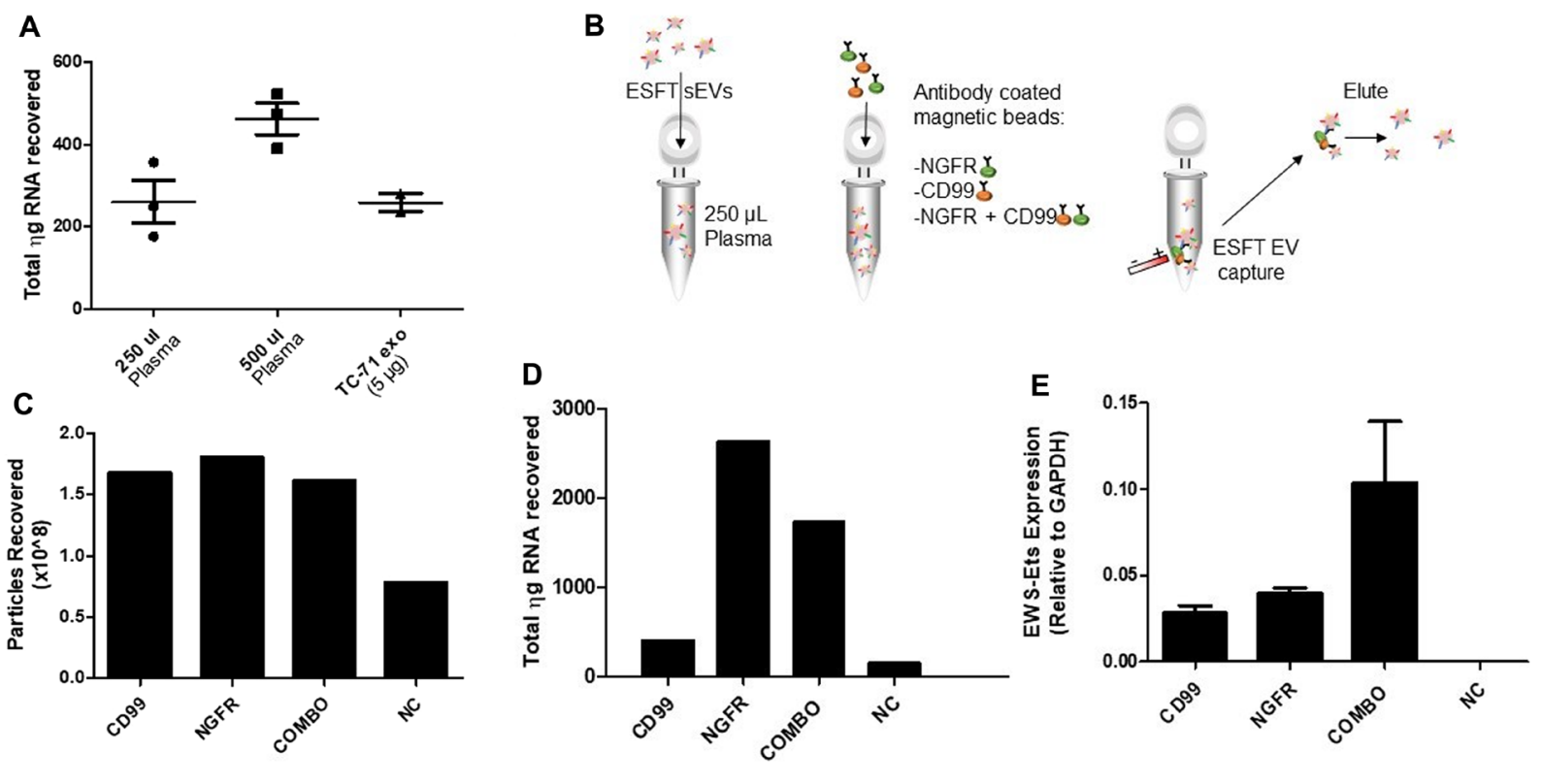

Figure 5: Development of an IP to enrich for ESFT-specific sEVs. (A) Amount of total RNA (in nanograms) recovered from sEVs isolated through ultracentrifugation from human plasma samples $(250 \mu \mathrm{L}$ or $500 \mu \mathrm{L})$ and from TC-71 derived sEVs as a control. (B) Schematic depicting the methodology of our IP strategy. (C-E) Particles recovered (C), total RNA (in ng) recovered (D), and the relative abundance of EWS-ETS transcripts (E) obtained from sEVs isolated from sEV-spiked plasma samples. EWS-ETS expression comparison utilizing CD99 versus NGFR versus CD99 + NGFR (COMBO) antibody cocktail for enrichment of ESFT sEVs. The COMBO demonstrated greater yield for increased enrichment of ESFT sEVs. 
Table 1: Patient information

\begin{tabular}{lcccc}
\hline ID\# & Age (Years) & Gender & EWSR FISH & Localized/Metastatic \\
\hline 1 & 16 & M & + & Metastatic \\
8 & 6 & F & + & Localized \\
9 & 9 & F & + & Metastatic \\
13 & 1.6 & F & + & Localized \\
14 & 6 & M & + & Metastatic \\
16 & 12 & F & + & Localized \\
18 & 15 & F & + & Localized \\
20 & 6 & F & + & Metastatic \\
23 & 16 & F & + & Metastatic \\
25 & 17 & M & + & Localized \\
026785 & 3 & N & NA & NA \\
236786 & 20 & M & NA & NA \\
026839 & 2 & F & NA & NA \\
026940 & 11 & F & NA & NA \\
027153 & 20 & M & NA & NA \\
027161 & 18 & M & NA \\
\hline
\end{tabular}

Ten (10) pediatric ESFT patients and 6 non-oncology pediatric patients were utilized for the analysis Ewing Sarcoma derived small extracellular vesicles (sEV).

of otherwise low frequency mutations, i.e., EWS-ETS, which is diagnostic of the disease. By initiating studies to develop a liquid-based biopsy we sought to improve the molecular tools for the diagnosis, detection, and disease monitoring of patients with ESFT.

The use of blood-based diagnostics, referred to as liquid biopsies, provides an opportunity improve diagnosis and to monitor disease states in real-time [46]. Circulating tumor cells (CTCs) and circulating tumor DNA (ctDNA) have been used clinically, but their diagnostic value is still limited for many cancer types based on sensitivity and specificity of the respective assays. For example, the heterogeneity and rarity of CTCs define the challenges of purifying an extremely small number of CTCs from a large number amount of other cells in a large blood volume (typically $7.5 \mathrm{~mL}$ ) [47]. In metastatic cancer setting, CTCs within the peripheral circulation occur at an estimated number of one CTC per $1 \times 10^{5-7}$ peripheral blood mononuclear cells [48]. Relevant to our studies, Benini et al. reported that CD99+ CTCs were detected in 4 of 23 peripheral ESFT using $10-\mathrm{mL}$ blood samples from patients age range of 13-32 years. ctDNA is another option being promoted as an alternative noninvasive method that overcome many difficulties related to tissue biopsy (e.g., spectrum of mutations limited to a single region of the tumor, serial sampling usually not feasible, etc.). Though significant progress has been accomplished in the field of ctDNA diagnostics, especially those based on next generation sequencing, serious limitation exists, given the vast majority of circulating DNA is primarily composed of normal cell free DNA (cfDNA) [49, 50].
Relevant to our studies, Shulman et al. utilizing an NGS hybrid capture assay and an ultra-low-pass whole-genome sequencing assay to detect ctDNA in a median of $2 \mathrm{~mL}$ of banked localized ESFT pediatric patient plasma from Children's Oncology Group (COG), demonstrated ctDNA in 53.3\% (41/77) of newly diagnosed patients [51]. Allegretti et al. demonstrated that EWS-FLI1 Type I and Type II rearrangements could be identified, regardless of patient-specific EWS-FLII DNA breakpoints in circulating tumor RNAs (ctRNAs) in 4 patients (1 metastatic and 3 localized) ranging in ages from $8-45$ years utilizing 1.8 $\mathrm{mLs}$ of plasma. Although the most frequent translocation partner of EWS is FLI1, with the common fusion joining EWS exon 7 in frame with FLII exon 6 (type 1 fusion), there are several other EWS-FLII type fusions, as well $5-10 \%$ of patients with ESFT have an EWS partner $E R G$. Our method focused on ESFT exo-proteins to immunoenrich tumor-associated circulating EVs for the subsequent detection of EWS-FLII Types I, II, and III and EWS-ERG fusion transcripts within pediatric patient plasma. ESFT is regarded as a malignancy of childhood and adolescence and thus rare in over the age of 40 , hence our focus on enrolling patients who consist of the majority of this disease population in this assay. A limitation in pediatric studies such as this is in part due to the incidence of ESFT in children and young adults within the United States and the volume of blood ethically and safely obtainable, hence we have utilized 10 pediatric patients in this study and were able to detect EWS-ETS fusion transcripts in both metastatic and localized subset of patients utilizing only $250 \mu \mathrm{L}$ of plasma. We have expanded and improved upon 
prior published accomplishments [52-54] by directly identifying ESFT sEV-associated protein biomarkers which enabled us to enrich for ESFT-specific sEVs $(\mathrm{AUC}=0.92)$ and concordantly detecting the EWS-ETS fusion transcript $(\mathrm{PPV}=1.00$ and $\mathrm{NPV}=0.67)$ from as little as $250 \mu \mathrm{l}$ of archival plasma samples. The approach by Benini et al. [55], Allegretti et al. [56], and our own circumvent the requirement to sequence patient-specific breakpoints, obtain long tumor DNA fragments from fresh tumor, and design patient-specific primer sets. Based on the above technical background, our clinically relevant assay could be applied to diagnose and potentially monitor ESFT patients during therapy and then off therapy for recurrence of disease.

In this first proteomic analysis of ESFT derived sEVs, we demonstrate the presence of 618 core enriched ESFT-sEV proteins, including ESFT associated proteins such as CD99/MIC2, caveolin, and GLG1 which have recently been proposed as markers for ESFT [57]. Among the top SEV biomarker candidates, we identified both HINT1 and NGFR (p75NTR). Previously, HINT1 was found to repress $\beta$-catenin-mediated transcription of Wnt target genes, and had been noted to be differentially expressed between localized and metastatic ESFT [44]. NGFR, also known as low-affinity nerve growth factor, a member of the tumor necrosis receptor family and has been implicated in the paracrine growth regulation of a number of neuronal as well as non-neuronal tumor types [58], such as prostate cancer, invasive ductal breast cancer, pancreatic carcinoma and malignant melanoma. NGFR is abundantly expressed during development but in adult organisms is known to be downregulated. However, the NGFR is re-expressed in conditions of increased neuronal cell death [59]. In a study done by Fanburg-Smith and Miettinen, non-neural mesenchymal tumors showed variable NGFR expression based on tumor type, with rhabdomyosarcoma demonstrating a $90 \%$ positivity of 94 cases, Ewing Sarcoma 32\% in 31 cases and extraskeletal osteosarcomas $23 \%$ in 13 cases [60]. Likewise, we observed over $80 \%$ of tissue samples positive for NGFR by IHC. Both CD99 and NGFR on subsequent analyses of the mass spectrometry sEV proteomics utilizing Proteome Discoverer v2.3.0.523 confirmed that both of these proteins are in the top $15 \%$ based on the respective MS1 data and PSM. In addition to ESFT-specific proteins, the detection of chimeric mRNAs transcribed from the EWSETS fusion genes are a valuable tool in the molecular diagnosis of ESFTs [61]. Overall the ESFT genome is genetically quiet with few genomic aberrations/mutations identified compared with most cancers [62-64]. In recent
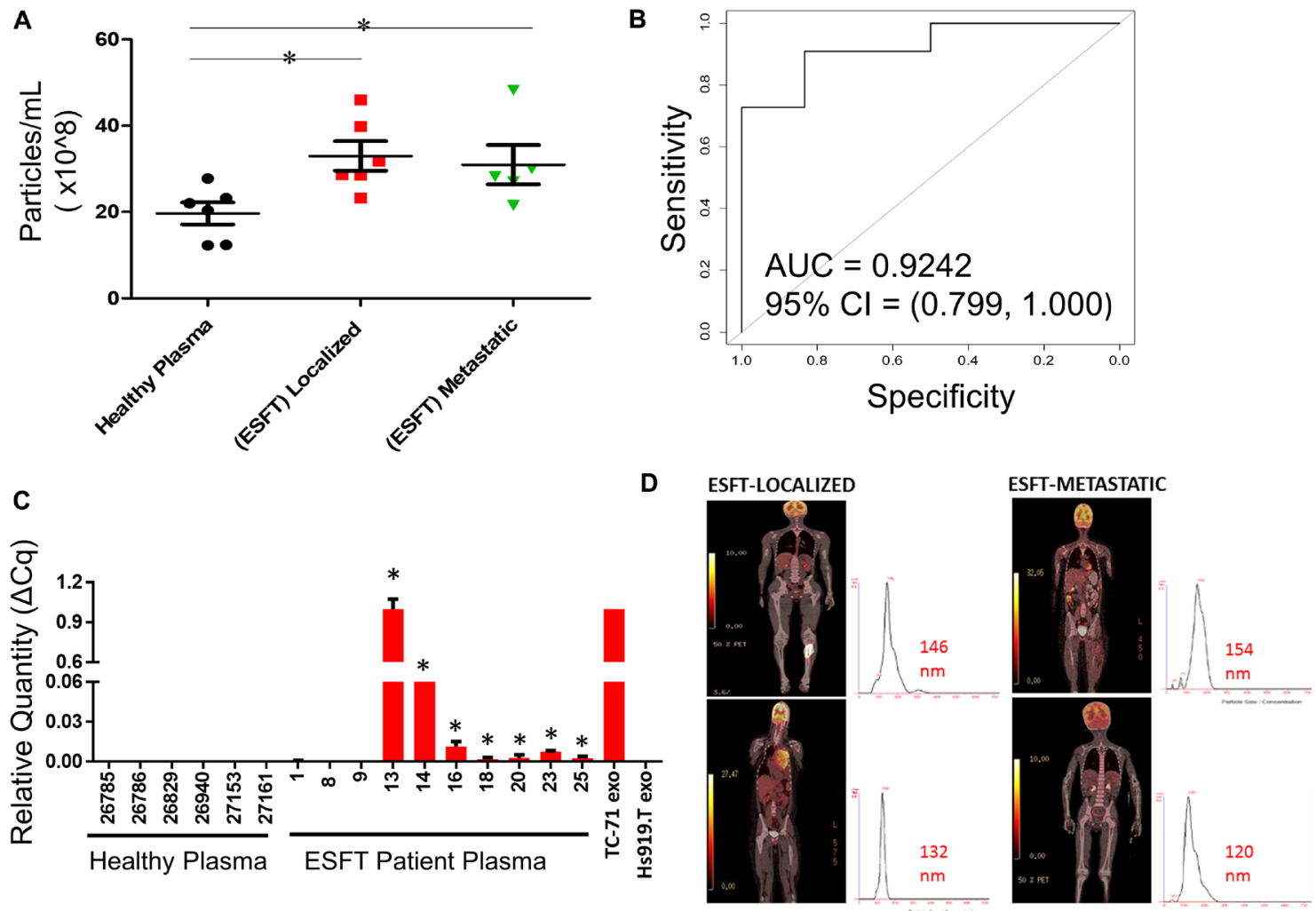

Figure 6: Enrichment of ESFT-sEVs from clinical ESFT plasma samples. (A) Total concentration of sEV's isolated from 250 $\mu \mathrm{L}$ of pediatric ESFT $(n=10)$ and healthy plasma $(n=6)$. (B) Area Under the Curve (AUC) analysis (A) of total sEV counts in cases and controls following immuno-enrichment. (C) Detection of the EWS-ETS transcript by qRT-PCR, ${ }^{*}$ denotes samples that crossed threshold and are considered positive, total of 7/10. cDNA from sEVs derived from TC-71 and Hs919.T cell lines served as positive and negative controls, respectively. (D) Localized and metastatic pediatric ESFT patient isolated sEVs demonstrating size threshold between 120-154 nm. 
years, it has become abundantly clear that the EWSETS rearrangements are the most important molecular determinant of tumorigenesis and progression of the ESFT [65]. This makes the identification of ESFT related proteomics and translocations in circulation particularly appealing because they are not likely to be lost during tumorigenesis and tumor progression. This current study is the first to identify the EWS-ETS transcript with high specificity in sEVs isolated from pediatric ESFT patient plasma samples by combining $\mathrm{sEV}$ proteomics and immuno-enrichment techniques.

The composition of sEVs is not sporadic in nature, suggestive that the incorporation of proteomic and RNA cargo into sEVs are a regulated process. Different forms of post translational modifications of proteins have been reported to occur in $\mathrm{sEVs}$, such modifications permit for protein versatility via influencing their activation state, subcellular localization, stability, and protein: protein interactions [66]. Certain aspects of post-translational modification have been shown to be integrated within the biopathway of sEV release. The generation and progression of many diseases have been associated with sEVmediated transport of misfolded disease-causing proteins as well [67]. Loading of proteins within sEVs because of protein damaging modifications such as oxidation [68] or misfolded proteins has also been well described [69]. Our results suggest that some proteins within ESFT derived-sEVs/exosomes, including EWS-ETS fusion proteins, are likely post-translationally modified prior to sorting. The presence of abundantly enriched proteins on the membrane of sEVs as well as those of modified proteins in sEVs offers an excellent opportunity to develop highly specific techniques for the isolation and identification of sEVs for biomarker utility; thus, offering an unprecedented opportunity to garner information for pediatric sarcomas in a non-invasive manner and help potentially design curative options that would further improve on the OS of these patients. A crucial challenge to our assay is in achieving absolute sensitivity, while avoiding any false positives. The latter is not a major issue given that EWS-ETS fusion are uncommon in other cancer types; however, the presence of the EWS-ETS transcript is as low as $<1$ copy $/ 10^{5} \mathrm{sEVs}$ as we have previously published [52], which is a likely reason for our inability to achieve $100 \%$ specificity with the current assay. There are several approaches that may further enhance aspects of our technique. Effective combinations of antibodies towards ESFT sEVs membrane-based antigens discovered through our proteomics alongside NGFR and CD99 for immunocapture, rather than dual-antibody approaches implemented within our study, may potentially further improve sEV isolation. Another approach would be to increase the volume of plasma input into our assay to $500-1,000 \mu \mathrm{L}$. Prior studies have demonstrated approximately $2.11 \%$ of the total RNA content within sEVs are mRNA fragments, while microRNAs are vastly enriched within these circulating extracellular vesicles. We are currently studying the utility of ESFT sEV miRNAs as biomarkers in conjunction with our EWS-ETS detection methodologies. By incorporating several markers for the detection and diagnosis of ESFT, a biomarker signature as such will further increase the sensitivity of this assay to enable the identification of even minimal residual disease presence during therapy and even post-therapy.

In order to further advance the development of sEVs as biomarkers in ESFT, our ongoing studies are integrating protein markers identified through our study into our prototypic microfluidic chip. As discussed above, we have recently demonstrated the quantitative measurement of EWS-FLI1 mRNA copy numbers in pPNET-derived sEVs [52]. Although a rare disease, development of this type of integrated assay could aid in the diagnosis of all members of the ESFT family. We are currently in process of developing of single microfluidic platform using our validated capture reagents to streamline the enrichment of tumor derived sEVs and quantitative measurement of EWS-ETS fusion transcripts in ESFT. Studies of ESFT tumor derived sEVs may reveal possible new important therapeutic targets, as well as perhaps yield RNAs prognostic for tumor aggressiveness and chemotherapeutic sensitivity allowing clinicians to better treat this pediatric malignancy.

\section{MATERIALS AND METHODS}

\section{Cell line and culture conditions}

Hs919. T, SK-ES-1, RD-ES were purchased from the American Type Culture Collection. In addition, TC-71, COG-E-352, and CHLA-258 cell lines were obtained from the Children's Oncology Group (COG). All cell line identities were confirmed by short tandem repeat profiling via the Clinical Molecular Oncology Laboratory at KUMC and the causative mutations in ESFT were confirmed by FISH utilizing the EWSR1 break apart probes for EWS translocation at Children's Mercy Clinical Genetics and Genomics Laboratories. All cell lines were cultured at $37^{\circ} \mathrm{C}$ under a $5 \%$ humidified $\mathrm{CO}_{2}$ atmosphere. TC-71, COG-E-352 and CHLA-258 cell lines were maintained in Iscoves Modified Dulbecco's Medium (IMDM), supplemented with L-glutamine (3 $\mathrm{mM})$, insulin, and transferrin ( $5 \mathrm{mg} / \mathrm{ml}$ each), selenium ( $5 \mathrm{ng} / \mathrm{ml}$ ), and $20 \%$ heat-inactivated exosome free FBS (whole medium). RD-ES cell line was maintained in RPMI 1640 with L-glutamine, supplemented with 15\% heat-inactivated exosome free FBS (whole medium). SKES-1 was maintained in McCoy's 5A with L-glutamine supplemented with $15 \%$ heat-inactivated exosome free FBS (whole medium). Hs919. T cells were maintained in DMEM with high glucose with L-glutamine, supplemented with $20 \%$ heat-inactivated exosome free FBS (whole medium). All cell lines were cultured in 
the presence of $10 \%$ penicillin streptomycin to prevent bacterial growth/contamination.

\section{SEV isolation from conditioned medium of cultured cells}

ESFT cell lines were grown and cultured in five T175 $\mathrm{cm}^{2}$ flasks containing $10 \%$ exosome-free FBS medium for 48-72 hours until cellular sub-confluency of $\sim 70 \%$ was reached. The benign osteoid osteoma control cell line, Hs919. T was cultured in five $T 175 \mathrm{~cm}^{2}$ flasks in $20 \%$ exosome-free FBS media for 168-240 hours until cellular sub-confluency of $\sim 60-70 \%$. Media were collected and immediately centrifuged at 2,500 rpms for 5 minutes to eliminate cellular debris. A total of $150 \mathrm{~mL}$ of conditioned medium was collected and ultra-centrifuged at $4^{\circ} \mathrm{C}$ for 45 minutes at $8,700 \mathrm{rpm}(10,000 \times \mathrm{g})$. The supernatant was then collected and ultracentrifuged again at $4^{\circ} \mathrm{C}$ for 75 minutes at $28,800 \mathrm{rpms}(110,000 \times \mathrm{g})$. sEV pellets were washed with PBS and were collected by ultracentrifugation at $4^{\circ} \mathrm{C}$ for 60 minutes at $35,800 \mathrm{rpms}$ in Beckman Coulter Quik-Seal Centrifuge Tubes. Finally, each sEV pellet was resuspended in $50-100 \mu 1$ of PBS based on pellet size and then stored at $-80^{\circ} \mathrm{C}$.

\section{Nanoparticle tracking analysis (NTA)}

Size and concentration of isolated and purified cell line derived SEV analysis was via the NanoSight LM10 (NanoSight Ltd., Minton Park, Amesbury, UK). A 1:1500 sEV pellet dilution in PBS was used for this analysis. NTA is a system for particle size analysis ranging from $30-1,000 \mathrm{~nm}$, with lower detectable limits dependent on the refractive index of the nanoparticles. This technique combines laser light scattering microscopy with a chargecoupled device (CCD) camera, enabling the visualization and recording of nanoparticles within solution.

\section{SEV proteomics analysis}

ESFT cell line derived sEV were reduced with 0.1 M DTT for $60^{\circ} \mathrm{C}$ for 30 min prior to dilution into FilterAided Sample Preparation (FASP) buffer ( $8 \mathrm{M}$ urea, 0.1 $\mathrm{M}$ Tris-HCl $\mathrm{pH}$ 8.5) and transferred to a Microcon-10 (EMD Millipore, Billerica, MA, USA) 10kDa centrifugal for trypsinization by the FASP method [70, 71]. Tryptic digests were trap cleaned using C18 PROTO $^{\text {TM }}$ Ultra MicroSpin columns (Nest Group, Inc, Southborough, MA) then lyophilized and redissolved into $2 \%$ acetonitrile $/ 0.1 \%$ formic acid prior to LCMS analysis. Tryptic peptides were separated using an EASY n-LC (Thermo) UHPLC system and a $360 \mu \mathrm{m}$ OD $\times 100 \mu \mathrm{m}$ ID fused silica tip packed with $10 \mathrm{~cm}$ of Jupiter $5 \mu \mathrm{m} \mathrm{C} 18300 \AA$ material (Phenomenex, Torrance, CA, USA). Following injection of the sample onto the column, separation was accomplished with a 75 min linear gradient from $2 \%$ acetonitrile to $40 \%$ acetonitrile in $0.1 \%$ formic acid. The eluate was introduced into the LTQ-Velos-Orbitrap ELITE + ETD mass spectrometer using a Nanospray Flex source (ThermoElectron, Waltham, MA, USA). An Orbitrap Elite - ETD mass spectrometer (ThermoElectron) was used to collect data from the LC eluate. An Nth Order Double Play with ETD Decision Tree method was created in Xcalibur v2.2. Scan event one obtained an FTMS MS1 scan (normal mass range; 60,000 resolution, full scan type, positive polarity, profile data type) for the range $300-2000 \mathrm{~m} / \mathrm{z}$. Scan event two obtained ITMS MS2 scans (normal mass range, rapid scan rate, centroid data type) on up to ten peaks that had a minimum signal threshold of 20,000 counts from scan event one. Each sample was injected twice yielding essentially a technical replicate, to aid with observation and ID of low abundant proteins. A decision tree was used to determine whether CID or ETD activation was used. An ETD scan was triggered if any of the following held: an ion had charge state 3 and $\mathrm{m} / \mathrm{z}$ less than 650, an ion had charge state 4 and $\mathrm{m} / \mathrm{z}$ less than 900 , an ion had charge state 5 and $\mathrm{m} / \mathrm{z}$ less than 950 , or an ion had charge state greater than 5; a CID scan was triggered in all other cases. The lock mass option was enabled ( $0 \%$ lock mass abundance) using the 371.101236 $\mathrm{m} / \mathrm{z}$ polysiloxane peak as an internal calibrant. Initially data dependent spectra search was directed by Proteome Discoverer v1.3.0.330 (ThermoElectron) using Mascot v2.1 and Sage-N Sorcerer Sequest algorithms and the UniprotKB Homo sapiens reference proteome canonical and isoform sequences (7/10/2013 version). Search parameters included: variable methionine oxidation, fixed cysteine carbamidomethylation, up to 2 missed tryptic cleavages, $50 \mathrm{ppm}$ precursor error for MS1 Orbitrap FTMS data, 0.8 Da error for CID-based MS2 LTQ data and 1.2Da error for ETD-based MS2 data. In order to estimate the false discovery rate, a decoy database was generated from this database with the program decoy. pl (from http://www.matrixscience.com/).

The ESFT cell line derived sEV mass spectrometry proteomics data was evaluated by Proteome Discoverer v2.3.0.523 (ThermoElectron) for imputation, matchbetween-runs, normalization steps, and protein modification for carbamylation to address effects of urea introduced during the FASP protocol. Search parameters included: variable methionine oxidation, fixed cysteine carbamidomethylation, up to 2 missed tryptic cleavages, $50 \mathrm{ppm}$ precursor error for MS1 Orbitrap FTMS data, $0.8 \mathrm{Da}$ error for CID-based MS2 LTQ data and 1.2Da error for ETD-based MS2 data. Technical duplicate data were searched and integrated as one sample. In order to estimate the false discovery rate, a decoy database was generated from this database with the program decoy. $\mathrm{pl}$ (from http://www.matrixscience.com/). For samples with peptides not confidently identified via Proteome Discoverer v2.3.0.523 utilized mass tolerance windows and chromatographic alignments to determine peptide 
presence. With presence of an $\mathrm{m} / \mathrm{z}$ signal, area of the ion was extracted and utilized to populate missing values. For runs without $\mathrm{m} / \mathrm{z}$ values within the mass accuracy window nor chromatographic retention time then no value was recorded. MS1 area values for peptides with high confident MS2 data were represented as high signal levels (green values). MS1 area values for peptide features that match within mass accuracy tolerance and retention time tolerance to high confidence data in a separate LCMS run were represented as peak found but not sufficient for MS2 validation (yellow values). For non-detectable proteins without sufficient information to assign high confidence spectra (green value) nor with mass accuracy or retention time tolerances (yellow data), no signal or red value was assigned.

\section{Bioinformatics analysis}

Initial analysis of mass spectrometry proteomics dependent spectra directed by Proteome Discoverer v1.3.0.330 identified 1082 proteins. Protein identity and quantitative data were exported for further statistical and bioinformatic analysis. The list of differentially enriched proteins in $\mathrm{SEV}$ were submitted to identify the enrichment of biological processes in SEV according their gene ontology annotation extracted from the UniProt database. Qualitative data generated from proteomic mass spectrometric analysis were analyzed using Ingenuity Pathway Analysis (IPA; Ingenuity System, Redwood City, CA, USA; http://www.ingenuity.com), Plasma Proteome Database (http://www.plasmaproteomedatabase.org/) and Exocarta Vesiculopedia to identify potential biomarker sEV signatures and their application in pediatric sarcomas.

Quantitative data from proteomic mass spectrometric analysis were analyzed using the $\mathrm{R}$ statistical programming language (http://cran.r-project. org) using $\mathrm{R}$ version 3.6.1. For each biological replicate, there were two technical replicates in which total spectral counts were available. If the total spectral count was " 0 " or "NA" it was replaced with a value of " 0.5 ". These total spectrum counts were averaged for each pair of technical replicates and then $\log 2$ transformed for analysis. Protein expression data collected from ESFT cell line derived sEVs from EWS-FLI1 Type I fusion (TC-71, $n=2$ ), EWS-FLI1 Type II fusion (SK-ES-1, $n=1$ and RD-ES, $n=1$ ), EWS-FLI1 Type III fusion (CHLA-258, $n=2$ ), and EWS-ERG fusion (COG-E-352, $n=1$ ) was $\log _{2}$ transformed prior to analysis. Due to the lack of biological replicates for some of the cell lines, one-way analysis of variance (ANOVA) models were fit independently to each of the proteins and linear contrasts were used to test the comparisons of interest. $P$-values and $95 \%$ confidence intervals were calculated for each of the linear contrasts. Volcano plots were generated for each comparison of interest with the difference in mean $\log _{2}$ (expression) plotted versus the $-\log _{10}$ ( $p$-value).
To identify disrupted biological pathways between EWS-FLI1 versus EWS-ERG cell lines, a gene set enrichment analysis (GSEA) was implemented in R using the package gage [72]. Protein expression data (total spectrum count) from the EWS-FLI1 $(n=6)$ and EWS-ERG $(n=1)$ cell lines were used as the basis of comparison. The predefined gene set used was derived from KEGG pathways and stored in the 'kegg.gs' data set in the gage package.

\section{SDS-PAGE and western blot analysis}

sEV samples and cell lysates were prepared in radioimmunoprecipitation assay (RIPA) buffer and separated by adding $40 \mu \mathrm{g}$ protein on $7 \%, 10 \%$, or $4-20 \%$ MiniPROTEAN $^{\circledR}$ TGX $^{\text {TM }}$ Precast Gels, (BioRad, Hercules, CA, USA). The completed gels were transferred to a supported nitrocellulose membrane (BioRad). The membranes were blocked with 5\% non-fat milk for one hour with gentle rocking. Membranes were incubated with primary antibodies overnight and then washed thrice for 10 minutes before addition of HRP conjugated anti-rabbit or anti-mouse secondary antibody (BioRad) for 1 hour. Membranes were subsequently washed and treated with ECL Western Blotting Substrate (Fisher Scientific) according to manufacturer's instructions and antibodies used were either rabbit or mouse Isotype (Supplementary Table 8).

\section{Immuno-pulldown and analysis of specific population of plasma-derived SEVs}

Immuno-pulldown of sEVs required two overnight 18 to 20-hour incubation steps. Day 1 consisted of Dynabead $^{\mathrm{TM}}$ washes and immobilization of antibodies. Dynabead Wash-1,000 $\mu \mathrm{g}(100 \mu \mathrm{L})$ of Dynabeads ${ }^{\mathrm{TM}}$ M-270 Streptavidin beads were washed three times in PBS Buffer. Immobilization of Antibodies- $10 \mu \mathrm{g}$ of biotinylated antibody were incubated with beads overnight with 1,000 $\mu \mathrm{g}$ of Dynabeads. Day 2 consisted of Dynabead washes and immobilization of exosomal proteins. Dynabead Washantibody coated beads were separated by use of a magnet. Supernatants were removed and subsequently beads were washed 4-5 times with PBS containing 0.1\% BSA. Antibody coated beads were then resuspended in $100 \mu \mathrm{L}$. A total of $50 \mu \mathrm{L}$ of the antibody coated bead suspension was placed in $250 \mu \mathrm{L}$ of plasma and incubated overnight to immobilize sEV membranous proteins. Day 3 consisted antibody coated beads exosomal conjugate washes with PBS and then separation with Exoquick ${ }^{\mathrm{TM}}$ Exosome Precipitation solution (System Biosciences) with magnet to dissociate the biotin-streptavidin bond.

\section{qRT-PCR of exoEWS-ETS fusion transcripts}

RNA isolated from cells and sEV samples were by utilizing the miRNeasy kit (Qiagen) in combination with phase lock tubes (5-Prime) according to the 
manufacturer protocols for total RNA isolation. Prior to RNA isolation, sEV pellets were treated with RNAse A (Thermo Fisher) at a concentration of $5 \mu \mathrm{g} / \mathrm{ml}$ for 30 minutes at $4^{\circ} \mathrm{C}$. cDNA was made from 50-100 ng total RNA and using SuperScript IV VILO cDNA synthesis kit (Thermo Fisher). cDNA from cell lines was diluted (1/4) with TE buffer. cDNA from sEV samples was not diluted further. For detection of the EWS-FLI1 types I, II, and III transcript we used the forward primer EWSF 5'-GCCAAGCTCCAAGTCAATATATAGCCAACAG-3', and the reverse primer FLIR 5'-GGGCCGTTGCTCTGTATTCTTACTG-3'. $\quad$ For the detection of EWS-Erg fusion transcript we utilized the EWSF forward primer with the reverse primer ERGR1 5'-GAGTTGGAGCTGTCCGACAGG-3'. For each transcript we used a Fam-labeled probe 5'-GCAGCAGCTACGGGCAGCAG-3'. All primers and probes were manufactured by IDT. qRT-PCR assays were run on a CFX96 (BioRad) in volumes of $20 \mu \mathrm{l}$ according to the following conditions: $50^{\circ} \mathrm{C}, 3$ minutes; $95^{\circ} \mathrm{C}, 10$ minutes; then 40 cycles of $95^{\circ} \mathrm{C}, 15$ seconds; and $54^{\circ} \mathrm{C}, 60$ seconds.

\section{Pediatric sarcoma patient biobanking}

The translational aspect of this study was conducted within the framework of a newly initiated Children's Mercy Kansas City IRB approved protocol (PI: G. Samuel, CMH IRB\# 13010015), which is an ongoing, prospective observational single-center study. Enrollment criteria were for patients less than 18 years of age with newly diagnosed pediatric sarcomas focused primarily on pediatric ESFT, Rhabdomyosarcoma and Osteosarcoma. Patients were recruited while either on the inpatient or outpatient setting of Children's Mercy Kansas City Department of Hematology Oncology and Bone Marrow Transplantation starting December 2012; recruitment is ongoing. All newly diagnosed and recurrent patients were enrolled; a few patients actively undergoing therapy were also enrolled at the time of initiation of this protocol. The majority of enrolled patients had not received chemotherapy or radiotherapy prior to study inclusion. Parents of eligible patients aged below 7 years gave written informed consent. Patients 7 years and above were also asked to provide written assent. Samples were collected prior to initiation of therapy, immediately prior to each cycle of therapy and then every three months off therapy for the first two years only. At enrollment, data on medical history were collected including demographic and clinical details of tumor site, histology and stage were documented. During the following observation period further blood samples were taken prior to each cycle of chemotherapy or prior to local control (surgery and/or radiation therapy).

\section{Statistical analysis}

All statistical analyses were performed using $\mathrm{R}$ (http://cran.r-project.org). A one-way analysis of variance model (ANOVA) was used to compare the number of EVs (particles per $\mathrm{mL}$ ) assessed in plasma samples collected from healthy control individuals $(n=6)$ and clinical pediatric ESFT patients $(n=10)$. Briefly, the number of EVs was modeled as the dependent variable, against a single independent variable representing the health status of the individual (e.g., healthy plasma, ESFT localized, and ESFT metastatic). Parameter estimates obtained from the one-way ANOVA model were used to construct specific contrasts, which included a comparison of the mean number of EVs between: ESFT (both localized and metastatic) versus healthy, localized ESFT versus healthy, metastatic ESFT versus healthy, and localized ESFT versus metastatic ESFT. The positive predictive value (PPV) and negative predictive value (NPV) for detecting the EWS-ETS fusion transcript were computed using epitests function in the R package epiR. Confidence intervals for the PPV and NPV were calculated using a previously described procedure [70].

\section{Abbreviations}

ESFT: Ewing Sarcoma Family of Tumors; sEVs: small Extracellular Vesicles; PNET: peripheral primitive neuroectodermal tumors; MVB: multivesicular bodies; EFS: Event Free Survival; OS: Overall survival; CTCs: Circulating tumor cells; ctDNA: circulating tumor DNA.

\section{Author contributions}

A.K.G. conceived and G.S., J.C, K.N., and S.A. helped to design the study. G.S., J.C., S.A., and M.M. developed the methodology. G.S., J.C., S.A., and M.M. carried out experiments and collected data. G.S., J.C., M.M., J.B.K., D.C.K., E.N., and V.S. analyzed, computed and interpreted the data. G.S., J.C., and A.K.G. wrote the manuscript. G.S., J.C, S.A, J.B.K., K.L., K.N, D.C.K., E.N., V.S., and A.K.G. reviewed and revised the manuscript. K.L. and A.K.G. provided administrative, technical and material support. A.K.G. supervised the study.

\section{ACKNOWLEDGMENTS}

The authors gratefully acknowledge Drs. Katherine Chastain and Joy Fulbright who assisted in the accrual and consenting process of patients diagnosed at Children's Mercy onto the pediatric sarcoma biobank protocol (CMH IRB\#13010015) and the Biospecimen Repository Core Facility (BRCF) staff at KU Medical Center for processing and banking sample. We acknowledge Dr. Linda Cooley (Division Director of the Clinical Genetics and Genomics Laboratories, Children's Mercy) for FISH analysis of ESFT cell lines. We acknowledge the support of the University of Kansas Cancer Center's BRCF and Biostatistics and Informatics Shared Resource (P30 CA168524); the Massman Family Endowed Fund for 
Ewing Sarcoma Research and Zachary Durand Endowed Fund for Ewing Sarcoma Research. Most importantly, we acknowledge the children and young adults who along with their families consented to enroll and participate in this IRB study at Children's Mercy Kansas City by providing clinical specimens.

\section{Ethics statement}

The studies have been approved by the appropriate institutional research ethics committee and have been performed in accordance with the ethical standards as laid down in the 1964 Declaration of Helsinki and its later amendments or comparable ethical standards. As part of a joint collaboration between Children's Mercy (CM) in Kansas City, MO, USA and the Biospecimen Repository Core Facility (BRCF) at KU Cancer Center (HSC \#5929, Director, A. Godwin, PI), a pediatric sarcoma protocol (CMH IRB\#13010015, Glenson Samuel, MD) has been established to obtain tumor and blood specimens from pediatric sarcoma patients undergoing treatment at $\mathrm{CM}$ and stored in the Pediatric Sarcoma Biobank within the BRCF.

\section{Consent of publication}

Informed consent was obtained from all individual participants included in the study. Pediatric sarcoma pediatric patients are consented at Children's Mercy by a study member on the IRB approved study protocol either through the inpatient or outpatient oncology setting. Consents obtained from parents, and if appropriate, assent is obtained from the patient. Copies of consents are made and given to family and patients. De-identified Ewing Sarcoma Family of Tumor patient specimens, diseasefree controls and their accompanying clinical data were handled in an anonymous (coded) fashion.

\section{CONFLICTS OF INTEREST}

Authors have no conflicts of interest to declare.

\section{FUNDING}

This work was supported in part by grants from the NIH (R33 CA214333) \& the NIGMS P20 GM130423 to A.K.G. and P20 GM113226 to M.L.M, the MCA Partners Advisory Board from Children's Mercy Hospital, The University of Kansas Cancer Center (to G.S. and A.K.G.), Alex Lemonade Stand (to G.S.), Braden's Hope for Childhood Cancer Foundation (to G.S. \& A.K.G.), Noah's Bandage Project (to G.S. \& A.K.G.), Hyundai Hope on Wheels (to G.S), the KU Cancer Center's Cancer Center Support Grant (P30 CA168524), and the Kansas Bioscience Authority Eminent Scholar Program (to A.K.G.). J.B.K. is the James Graham Brown
Foundation Chair in Proteomics. A.K.G. is the Chancellors Distinguished Chair in Biomedical Sciences Endowed Professor.

\section{REFERENCES}

1. Suva ML, Riggi N, Stehle JC, Baumer K, Tercier S, Joseph JM, Suva D, Clement V, Provero P, Cironi L, Osterheld MC, Guillou L, Stamenkovic I. Identification of cancer stem cells in Ewing's sarcoma. Cancer Res. 2009; 69:1776-81. https:// doi.org/10.1158/0008-5472.CAN-08-2242. [PubMed]

2. Esiashvili N, Goodman M, Marcus RB Jr. Changes in incidence and survival of Ewing sarcoma patients over the past 3 decades: Surveillance Epidemiology and End Results data. J Pediatr Hematol Oncol. 2008; 30:425-30. https://doi. org/10.1097/MPH.0b013e31816e22f3. [PubMed]

3. Paulussen M, Ahrens S, Burdach S, Craft A, DockhornDworniczak B, Dunst J, Fröhlich B, Winkelmann W, Zoubek A, Jürgens H, and European Intergroup Cooperative Ewing Sarcoma Studies. Primary metastatic (stage IV) Ewing tumor: survival analysis of 171 patients from the EICESS studies. European Intergroup Cooperative Ewing Sarcoma Studies. Ann Oncol. 1998; 9:275-81. [PubMed]

4. Bernstein M, Kovar H, Paulussen M, Randall RL, Schuck A, Teot LA, Juergens H. Ewing's sarcoma family of tumors: current management. Oncologist. 2006; 11:503-19. https:// doi.org/10.1634/theoncologist.11-5-503. [PubMed]

5. Barker LM, Pendergrass TW, Sanders JE, Hawkins DS. Survival after recurrence of Ewing's sarcoma family of tumors. J Clin Oncol. 2005; 23:4354-62. https://doi. org/10.1200/JCO.2005.05.105. [PubMed]

6. Womer RB, West DC, Krailo MD, Dickman PS, Pawel BR, Grier HE, Marcus K, Sailer S, Healey JH, Dormans JP, Weiss AR. Randomized controlled trial of intervalcompressed chemotherapy for the treatment of localized Ewing sarcoma: a report from the Children's Oncology Group. J Clin Oncol. 2012; 30:4148-54. https://doi. org/10.1200/JCO.2011.41.5703. [PubMed]

7. Grier HE, Krailo MD, Tarbell NJ, Link MP, Fryer CJ, Pritchard DJ, Gebhardt MC, Dickman PS, Perlman EJ, Meyers PA, Donaldson SS, Moore S, Rausen AR, et al. Addition of ifosfamide and etoposide to standard chemotherapy for Ewing's sarcoma and primitive neuroectodermal tumor of bone. N Engl J Med. 2003; 348:694-701. https://doi. org/10.1056/NEJMoa020890. [PubMed]

8. Rodriguez-Galindo C, Spunt SL, Pappo AS. Treatment of Ewing sarcoma family of tumors: current status and outlook for the future. Med Pediatr Oncol. 2003; 40:276-87. https:// doi.org/10.1002/mpo.10240. [PubMed]

9. Gobel V, Jurgens H, Etspuler G, Kemperdick H, Jungblut RM, Stienen U, Gobel U. Prognostic significance of tumor volume in localized Ewing's sarcoma of bone in children and adolescents. J Cancer Res Clin Oncol. 1987; 113:18791. https://doi.org/10.1007/bf00391442. [PubMed] 
10. Picci P, Bohling T, Bacci G, Ferrari S, Sangiorgi L, Mercuri M, Ruggieri P, Manfrini M, Ferraro A, Casadei R, Benassi MS, Mancini AF, Rosito P, et al. Chemotherapy-induced tumor necrosis as a prognostic factor in localized Ewing's sarcoma of the extremities. J Clin Oncol. 1997; 15:1553-9. https://doi.org/10.1200/JCO.1997.15.4.1553. [PubMed]

11. Bacci G, Ferrari S, Bertoni F, Rimondini S, Longhi A, Bacchini P, Forni C, Manfrini M, Donati D, Picci P. Prognostic factors in nonmetastatic Ewing's sarcoma of bone treated with adjuvant chemotherapy: analysis of 359 patients at the Istituto Ortopedico Rizzoli. J Clin Oncol. 2000; 18:4 11. https://doi.org/10.1200/JCO.2000.18.1.4. [PubMed]

12. Li S, Yang Q, Wang H, Wang Z, Zuo D, Cai Z, Hua Y. Prognostic significance of serum lactate dehydrogenase levels in Ewing's sarcoma: A meta-analysis. Mol Clin Oncol. 2016; 5:832-8. https://doi.org/10.3892/ mco.2016.1066. [PubMed]

13. Potratz J, Dirksen U, Jurgens H, Craft A. Ewing sarcoma: clinical state-of-the-art. Pediatr Hematol Oncol. 2012; 29:1-11. https://doi.org/10.3109/08880018.2011.622034. [PubMed]

14. Pinto A, Dickman P, Parham D. Pathobiologic markers of the ewing sarcoma family of tumors: state of the art and prediction of behaviour. Sarcoma. 2011; 2011:856190. https://doi.org/10.1155/2011/856190. [PubMed]

15. Biermann JS, Chow W, Reed DR, Lucas D, Adkins DR, Agulnik M, Benjamin RS, Brigman B, Budd GT, Curry WT, Didwania A, Fabbri N, Hornicek FJ, et al. NCCN Guidelines Insights: Bone Cancer, Version 2.2017. J Natl Compr Canc Netw. 2017; 15:155-67. [PubMed]

16. Harris $\mathrm{MH}$, DuBois $\mathrm{SG}$, Glade Bender JL, Kim A, Crompton BD, Parker E, Dumont IP, Hong AL, Guo D, Church A, Stegmaier K, Roberts CW, Shusterman S, et al. Multicenter Feasibility Study of Tumor Molecular Profiling to Inform Therapeutic Decisions in Advanced Pediatric Solid Tumors: The Individualized Cancer Therapy (iCat) Study. JAMA Oncol. 2016; 2:608-615. https://doi. org/10.1001/jamaoncol.2015.5689. [PubMed]

17. Lopez-Guerrero JA, Machado I, Scotlandi K, Noguera R, Pellin A, Navarro S, Serra M, Calabuig-Farinas S, Picci P, Llombart-Bosch A. Clinicopathological significance of cell cycle regulation markers in a large series of genetically confirmed Ewing's sarcoma family of tumors. Int J Cancer. 2011; 128:1139-50. https://doi.org/10.1002/ijc.25424. [PubMed]

18. Jahromi MS, Putnam AR, Druzgal C, Wright J, SprakerPerlman H, Kinsey M, Zhou H, Boucher KM, Randall RL, Jones KB, Lucas D, Rosenberg A, Thomas D, et al. Molecular inversion probe analysis detects novel copy number alterations in Ewing sarcoma. Cancer Genet. 2012; 205:391-404. https://doi.org/10.1016/j. cancergen.2012.05.012. [PubMed]

19. Properzi F, Logozzi M, Fais S. Exosomes: the future of biomarkers in medicine. Biomark Med. 2013; 7:769-78. https://doi.org/10.2217/bmm.13.63. [PubMed]
20. Keller S, Ridinger J, Rupp AK, Janssen JW, Altevogt P. Body fluid derived exosomes as a novel template for clinical diagnostics. J Transl Med. 2011; 9:86. https://doi. org/10.1186/1479-5876-9-86. [PubMed]

21. Atay S, Banskota S, Crow J, Sethi G, Rink L, Godwin AK. Oncogenic KIT-containing exosomes increase gastrointestinal stromal tumor cell invasion. Proc Natl Acad Sci U S A. 2014; 111:711-6. https://doi.org/10.1073/ pnas.1310501111. [PubMed]

22. He M, Crow J, Roth M, Zeng Y, Godwin AK. Integrated immunoisolation and protein analysis of circulating exosomes using microfluidic technology. Lab Chip. 2014; 14:3773-80. https://doi.org/10.1039/c4lc00662c. [PubMed]

23. Thery C, Zitvogel L, Amigorena S. Exosomes: composition, biogenesis and function. Nat Rev Immunol. 2002; 2:56979. https://doi.org/10.1038/nri855. [PubMed]

24. Theodoraki MN, Hoffmann TK, Whiteside TL. Separation of plasma-derived exosomes into $\mathrm{CD} 3(+)$ and CD3(-) fractions allows for association of immune cell and tumour cell markers with disease activity in HNSCC patients. Clin Exp Immunol. 2018; 192:271-83. https://doi.org/10.1111/ cei.13113. [PubMed]

25. Atay S, Wilkey DW, Milhem M, Merchant M, Godwin AK. Insights into the Proteome of Gastrointestinal Stromal Tumors-Derived Exosomes Reveals New Potential Diagnostic Biomarkers. Mol Cell Proteomics. 2018; 17:495-515. https://doi.org/10.1074/mcp.RA117.000267. [PubMed]

26. Frampton AE, Prado MM, Lopez-Jimenez E, FajardoPuerta AB, Jawad ZAR, Lawton P, Giovannetti E, Habib NA, Castellano L, Stebbing J, Krell J, Jiao LR. Glypican-1 is enriched in circulating-exosomes in pancreatic cancer and correlates with tumor burden. Oncotarget. 2018; 9:1900613. https://doi.org/10.18632/oncotarget.24873. [PubMed]

27. Melo SA, Luecke LB, Kahlert C, Fernandez AF, Gammon ST, Kaye J, LeBleu VS, Mittendorf EA, Weitz J, Rahbari N, Reissfelder C, Pilarsky C, Fraga MF, et al. Glypican-1 identifies cancer exosomes and detects early pancreatic cancer. Nature. 2015; 523:177-82. https://doi.org/10.1038/ nature14581. [PubMed]

28. Hornick NI, Huan J, Doron B, Goloviznina NA, Lapidus J, Chang BH, Kurre P. Serum Exosome MicroRNA as a Minimally-Invasive Early Biomarker of AML. Sci Rep. 2015; 5:11295. https://doi.org/10.1038/srep11295. [PubMed]

29. Noerholm M, Balaj L, Limperg T, Salehi A, Zhu LD, Hochberg FH, Breakefield XO, Carter BS, Skog J. RNA expression patterns in serum microvesicles from patients with glioblastoma multiforme and controls. BMC Cancer. 2012; 12:22. https://doi.org/10.1186/1471-2407-12-22. [PubMed]

30. Nazarenko I, Rana S, Baumann A, McAlear J, Hellwig A, Trendelenburg M, Lochnit G, Preissner KT, Zoller M. Cell surface tetraspanin Tspan8 contributes to molecular pathways of exosome-induced endothelial cell activation. Cancer Res. 2010; 70:1668-78. https://doi. org/10.1158/0008-5472.CAN-09-2470. [PubMed] 
31. Peinado H, Aleckovic M, Lavotshkin S, Matei I, Costa-Silva B, Moreno-Bueno G, Hergueta-Redondo M, Williams C, Garcia-Santos G, Ghajar C, Nitadori-Hoshino A, Hoffman $\mathrm{C}$, Badal $\mathrm{K}$, et al. Melanoma exosomes educate bone marrow progenitor cells toward a pro-metastatic phenotype through MET. Nat Med. 2012; 18:883-91. https://doi. org/10.1038/nm.2753. [PubMed]

32. Chaput N, Thery C. Exosomes: immune properties and potential clinical implementations. Semin Immunopathol. 2011; 33:419-40. https://doi.org/10.1007/s00281-010-0233-9. [PubMed]

33. Crow J, Atay S, Banskota S, Artale B, Schmitt S, Godwin AK. Exosomes as mediators of platinum resistance in ovarian cancer. Oncotarget. 2017; 8:11917-36. https://doi. org/10.18632/oncotarget.14440. [PubMed]

34. Staals RH, Pruijn GJ. The human exosome and disease. Adv Exp Med Biol. 2011; 702:132-42. https://doi. org/10.1007/978-1-4419-7841-7 11. [PubMed]

35. Schaeffer D, van Hoof A. Different nuclease requirements for exosome-mediated degradation of normal and nonstop mRNAs. Proc Natl Acad Sci U S A. 2011; 108:2366-71. https://doi.org/10.1073/pnas.1013180108. [PubMed]

36. Miller IV, Raposo G, Welsch U, Prazeres da Costa O, Thiel U, Lebar M, Maurer M, Bender HU, von Luettichau I, Richter GH, Burdach S, Grunewald TG. First identification of Ewing's sarcoma-derived extracellular vesicles and exploration of their biological and potential diagnostic implications. Biol Cell. 2013; 105:289-303. https://doi. org/10.1111/boc.201200086. [PubMed]

37. Ma Y, Baltezor M, Rajewski L, Crow J, Samuel G, Staggs VS, Chastain KM, Toretsky JA, Weir SJ, Godwin AK. Targeted inhibition of histone deacetylase leads to suppression of Ewing sarcoma tumor growth through an unappreciated EWS-FLI1/HDAC3/HSP90 signaling axis. J Mol Med (Berl). 2019; 97:957-72. https://doi.org/10.1007/ s00109-019-01782-0. [PubMed]

38. Thery C, Amigorena S, Raposo G, Clayton A. Isolation and characterization of exosomes from cell culture supernatants and biological fluids. Curr Protoc Cell Biol. 2006; Chapter 3:Unit 3.22. https://doi.org/10.1002/0471143030.cb0322s30. [PubMed]

39. Arraud N, Linares R, Tan S, Gounou C, Pasquet JM, Mornet S, Brisson AR. Extracellular vesicles from blood plasma: determination of their morphology, size, phenotype and concentration. J Thromb Haemost. 2014; 12:614-27. https://doi.org/10.1111/jth.12554. [PubMed]

40. Moreno-Gonzalo O, Fernandez-Delgado I, Sanchez-Madrid F. Post-translational add-ons mark the path in exosomal protein sorting. Cell Mol Life Sci. 2018; 75:1-19. https:// doi.org/10.1007/s00018-017-2690-y. [PubMed]

41. Chang KT, Goytain A, Tucker T, Karsan A, Lee CH, Nielsen TO, Ng TL. Development and Evaluation of a Pan-Sarcoma Fusion Gene Detection Assay Using the NanoString nCounter Platform. J Mol Diagn. 2018; 20:63-77. https:// doi.org/10.1016/j.jmoldx.2017.09.007. [PubMed]
42. Panther JG, Stewart RR, Teasdale PR, Bennett WW, Welsh DT, Zhao H. Titanium dioxide-based DGT for measuring dissolved $\mathrm{As}(\mathrm{V}), \mathrm{V}(\mathrm{V}), \mathrm{Sb}(\mathrm{V}), \mathrm{Mo}(\mathrm{VI})$ and $\mathrm{W}(\mathrm{VI})$ in water. Talanta. 2013; 105:80-6. https://doi.org/10.1016/j. talanta.2012.11.070. [PubMed]

43. Anderson NL, Anderson NG. The human plasma proteome: history, character, and diagnostic prospects. Mol Cell Proteomics. 2002; 1:845-67. https://doi.org/10.1074/mcp. r200007-mcp200. [PubMed]

44. Schaefer KL, Eisenacher M, Braun Y, Brachwitz K, Wai DH, Dirksen U, Lanvers-Kaminsky C, Juergens H, Herrero D, Stegmaier S, Koscielniak E, Eggert A, Nathrath M, et al. Microarray analysis of Ewing's sarcoma family of tumours reveals characteristic gene expression signatures associated with metastasis and resistance to chemotherapy. Eur J Cancer. 2008; 44:699-709. https://doi.org/10.1016/j. ejca.2008.01.020. [PubMed]

45. Pant S, Hilton H, Burczynski ME. The multifaceted exosome: biogenesis, role in normal and aberrant cellular function, and frontiers for pharmacological and biomarker opportunities. Biochem Pharmacol. 2012; 83:1484-94. https://doi.org/10.1016/j.bcp.2011.12.037. [PubMed]

46. Siravegna G, Mussolin B, Buscarino M, Corti G, Cassingena A, Crisafulli G, Ponzetti A, Cremolini C, Amatu A, Lauricella C, Lamba S, Hobor S, Avallone A, et al. Clonal evolution and resistance to EGFR blockade in the blood of colorectal cancer patients. Nat Med. 2015; 21:827. https://doi.org/10.1038/nm0715-827b. [PubMed]

47. van de Stolpe A, Pantel K, Sleijfer S, Terstappen LW, den Toonder JM. Circulating tumor cell isolation and diagnostics: toward routine clinical use. Cancer Res. 2011; 71:5955-60. https://doi.org/10.1158/0008-5472.CAN-11-1254. [PubMed]

48. Ross AA, Cooper BW, Lazarus HM, Mackay W, Moss TJ, Ciobanu N, Tallman MS, Kennedy MJ, Davidson NE, Sweet D. Detection and viability of tumor cells in peripheral blood stem cell collections from breast cancer patients using immunocytochemical and clonogenic assay techniques. Blood. 1993; 82:2605-10. [PubMed]

49. Wang R, Li X, Zhang H, Wang K, He J. Cell-free circulating tumor DNA analysis for breast cancer and its clinical utilization as a biomarker. Oncotarget. 2017; 8:75742-55. https://doi.org/10.18632/oncotarget.20608. [PubMed]

50. Ziegler A, Zangemeister-Wittke U, Stahel RA. Circulating DNA: a new diagnostic gold mine? Cancer Treat Rev. 2002; 28:255-71. [PubMed]

51. Shulman DS, Klega K, Imamovic-Tuco A, Clapp A, Nag A, Thorner AR, Van Allen E, Ha G, Lessnick SL, Gorlick R, Janeway KA, Leavey PJ, Mascarenhas L, et al. Detection of circulating tumour DNA is associated with inferior outcomes in Ewing sarcoma and osteosarcoma: a report from the Children's Oncology Group. Br J Cancer. 2018; 119:615-21. https://doi.org/10.1038/s41416-018-0212-9. [PubMed]

52. Zhang P, Crow J, Lella D, Zhou X, Samuel G, Godwin AK, Zeng Y. Ultrasensitive quantification of tumor mRNAs in 
extracellular vesicles with an integrated microfluidic digital analysis chip. Lab Chip. 2018; 18:3790-801. https://doi. org/10.1039/c81c01071d. [PubMed]

53. Crow J, Samuel G, Godwin AK. Beyond tumor mutational burden: potential and limitations in using exosomes to predict response to immunotherapy. Expert Rev Mol Diagn. 2019; 19:1079-88. https://doi.org/10.1080/14737159.2020. 1688144. [PubMed]

54. Zhang P, Samuel G, Crow J, Godwin AK, Zeng Y. Molecular assessment of circulating exosomes toward liquid biopsy diagnosis of Ewing sarcoma family of tumors. Transl Res. 2018; 201:136-53. https://doi.org/10.1016/j. trs1.2018.05.007. [PubMed]

55. Benini S, Gamberi G, Cocchi S, Garbetta J, Alberti L, Righi A, Gambarotti M, Picci P, Ferrari S. Detection of circulating tumor cells in liquid biopsy from Ewing sarcoma patients. Cancer Manag Res. 2018; 10:49-60. https://doi. org/10.2147/CMAR.S141623. [PubMed]

56. Allegretti M, Casini B, Mandoj C, Benini S, Alberti L, Novello M, Melucci E, Conti L, Covello R, Pescarmona E, Milano GM, Annovazzi A, Anelli V, et al. Precision diagnostics of Ewing's sarcoma by liquid biopsy: circulating EWS-FLI1 fusion transcripts. Ther Adv Med Oncol. 2018; 10:1758835918774337. https://doi. org/10.1177/1758835918774337. [PubMed]

57. Baldauf MC, Orth MF, Dallmayer M, Marchetto A, Gerke JS, Rubio RA, Kiran MM, Musa J, Knott MML, Ohmura S, Li J, Akpolat N, Akatli AN, et al. Robust diagnosis of Ewing sarcoma by immunohistochemical detection of super-enhancerdriven EWSR1-ETS targets. Oncotarget. 2017; 9:1587-601. https://doi.org/10.18632/oncotarget.20098. [PubMed]

58. Miknyoczki SJ, Klein-Szanto AJ, Ruggeri BA. Neurotrophin-Trk receptor interactions in neoplasia: a possible role in interstitial and perineural invasion in ductal pancreatic cancer. Crit Rev Oncog. 1996; 7:89-100. [PubMed]

59. Dechant G, Barde YA. The neurotrophin receptor p75(NTR): novel functions and implications for diseases of the nervous system. Nat Neurosci. 2002; 5:1131-6. https:// doi.org/10.1038/nn1102-1131. [PubMed]

60. Fanburg-Smith JC, Miettinen M. Low-affinity nerve growth factor receptor (p75) in dermatofibrosarcoma protuberans and other nonneural tumors: a study of 1,150 tumors and fetal and adult normal tissues. Hum Pathol. 2001; 32:97683. https://doi.org/10.1053/hupa.2001.27602. [PubMed]

61. Delattre O, Zucman J, Melot T, Garau XS, Zucker JM, Lenoir GM, Ambros PF, Sheer D, Turc-Carel C, Triche TJ, Aurias A, Thomas G. The Ewing family of tumors--a subgroup of small-round-cell tumors defined by specific chimeric transcripts. N Engl J Med. 1994; 331:294-9. https://doi.org/10.1056/NEJM199408043310503. [PubMed]
62. Brohl AS, Solomon DA, Chang W, Wang J, Song Y, Sindiri S, Patidar R, Hurd L, Chen L, Shern JF, Liao H, Wen X, Gerard J, et al. The genomic landscape of the Ewing Sarcoma family of tumors reveals recurrent STAG2 mutation. PLoS Genet. 2014; 10:e1004475. https://doi. org/10.1371/journal.pgen.1004475. [PubMed]

63. Tirode F, Surdez D, Ma X, Parker M, Le Deley MC, Bahrami A, Zhang Z, Lapouble E, Grossetête-Lalami S, Rusch M, Reynaud S, Rio-Frio T, Hedlund E, et al, and St. Jude Children's Research Hospital-Washington University Pediatric Cancer Genome Project and the International Cancer Genome Consortium. Genomic landscape of Ewing sarcoma defines an aggressive subtype with co-association of STAG2 and TP53 mutations. Cancer Discov. 2014; 4:1342-53. https://doi.org/10.1158/2159-8290.CD-14-0622. [PubMed]

64. Crompton BD, Stewart C, Taylor-Weiner A, Alexe G, Kurek KC, Calicchio ML, Kiezun A, Carter SL, Shukla SA, Mehta SS, Thorner AR, de Torres C, Lavarino C, et al. The genomic landscape of pediatric Ewing sarcoma. Cancer Discov. 2014; 4:1326-41. https://doi.org/10.1158/21598290.CD-13-1037. [PubMed]

65. Kovar H, Ban J, Pospisilova S. Potentials for RNAi in sarcoma research and therapy: ewing's sarcoma as a model. Semin Cancer Biol. 2003; 13:275-81. [PubMed]

66. Moreno-Gonzalo O, Villarroya-Beltri C, Sanchez-Madrid F. Post-translational modifications of exosomal proteins. Front Immunol. 2014; 5:383. https://doi.org/10.3389/ fimmu.2014.00383. [PubMed]

67. Xiao T, Zhang W, Jiao B, Pan CZ, Liu X, Shen L. The role of exosomes in the pathogenesis of Alzheimer' disease. Transl Neurodegener. 2017; 6:3. https://doi.org/10.1186/ s40035-017-0072-x. [PubMed]

68. Anand S, Samuel M, Kumar S, Mathivanan S. Ticket to a bubble ride: Cargo sorting into exosomes and extracellular vesicles. Biochim Biophys Acta Proteins Proteom. 2019; 1867:140203. https://doi.org/10.1016/j.bbapap.2019.02.005. [PubMed]

69. Pink D, Donnelier J, Lewis J, Braun JEA. Cargo-Loading of Misfolded Proteins into Extracellular Vesicles: The Role of J Proteins. bioRxiv. 2018; 310219. https://doi. org/10.1101/310219.

70. Collett D. Modelling Binary Data. 2002.

71. Wisniewski JR, Zougman A, Nagaraj N, Mann M. Universal sample preparation method for proteome analysis. Nat Methods. 2009; 6:359-62. https://doi.org/10.1038/nmeth.1322. [PubMed]

72. Luo W, Friedman MS, Shedden K, Hankenson KD, Woolf PJ. GAGE: generally applicable gene set enrichment for pathway analysis. BMC Bioinformatics. 2009; 10:161. https://doi.org/10.1186/1471-2105-10-161. [PubMed] 